\title{
Automatic Diagnosis and Correction of Logical Errors for Functional Programming Assignments
}

\author{
JUNHO LEE, Korea University, Republic of Korea \\ DOWON SONG, Korea University, Republic of Korea \\ SUNBEOM SO, Korea University, Republic of Korea \\ HAKJOO $\mathrm{OH}^{*}$, Korea University, Republic of Korea
}

\begin{abstract}
We present FIxML, a system for automatically generating feedback on logical errors in functional programming assignments. As functional languages have been gaining popularity, the number of students enrolling functional programming courses has increased significantly. However, the quality of feedback, in particular for logical errors, is hardly satisfying. To provide personalized feedback on logical errors, we present a new errorcorrection algorithm for functional languages, which combines statistical error-localization and type-directed program synthesis enhanced with components reduction and search space pruning using symbolic execution. We implemented our algorithm in a tool, called FixML, and evaluated it with 497 students' submissions from 13 exercises, including not only introductory but also more advanced problems. Our experimental results show that our tool effectively corrects various and complex errors: it fixed $43 \%$ of the 497 submissions in 5.4 seconds on average and managed to fix a hard-to-find error in a large submission, consisting of 154 lines. We also performed user study with 18 undergraduate students and confirmed that our system actually helps students to better understand their programming errors.
\end{abstract}

CCS Concepts: • Software and its engineering $\rightarrow$ Automatic programming; Functional languages;

Additional Key Words and Phrases: Automated Program Repair, Program Synthesis

ACM Reference Format:

Junho Lee, Dowon Song, Sunbeom So, and Hakjoo Oh. 2018. Automatic Diagnosis and Correction of Logical Errors for Functional Programming Assignments. Proc. ACM Program. Lang. 2, OOPSLA, Article 158 (November 2018), 30 pages. https://doi.org/10.1145/3276528

\section{INTRODUCTION}

Motivation. The motivation for this work originated from an undergraduate course on functional programming taught by the authors over the last few years. As functional languages have been gaining popularity, the number of students enrolling the course has increased significantly. The quality of feedback, however, hardly satisfied the increased demands. Because most students have no experience in functional languages, they often have more difficulty with various programming errors than learning other languages such as Java or Python. However, assisting students to resolve

${ }^{*}$ Corresponding author

Authors' addresses: Junho Lee, junho_lee@korea.ac.kr, Department of Computer Science and Engineering, Korea University, 145, Anam-ro, Sungbuk-gu, Seoul, 02841, Republic of Korea; Dowon Song, dowon_song@korea.ac.kr, Department of Computer Science and Engineering, Korea University, 145, Anam-ro, Sungbuk-gu, Seoul, 02841, Republic of Korea; Sunbeom So, sunbeom_so@korea.ac.kr, Department of Computer Science and Engineering, Korea University, 145, Anam-ro, Sungbukgu, Seoul, 02841, Republic of Korea; Hakjoo Oh, hakjoo_oh@korea.ac.kr, Department of Computer Science and Engineering, Korea University, 145, Anam-ro, Sungbuk-gu, Seoul, 02841, Republic of Korea.

This work is licensed under a Creative Commons Attribution-NonCommercial-ShareAlike 4.0 International License.

(c) 2018 Copyright held by the owner/author(s).

2475-1421/2018/11-ART158

https://doi.org/10.1145/3276528

Proc. ACM Program. Lang., Vol. 2, No. OOPSLA, Article 158. Publication date: November 2018. 
those errors is challenging. In particular, providing personalized feedback on logical errors was most difficult as it requires instructors to manually figure out the cause of the errors, unlike syntax or type errors for which a wealth of tool support already exists [Chen and Erwig 2014; Lerner et al 2007; Pavlinovic et al. 2014, 2015; Seidel et al. 2017; Wu et al. 2017; Wu and Chen 2017; Zhang et al. 2017].

Providing simple, non-personalized feedback on logical errors was not much helpful for students. Since it is hard to give advice on a one-to-one basis to many students, one standard way of giving feedback on logical errors is to provide failing testcases. Unfortunately, debugging is a time-consuming and error-prone task even for the experts on programming, so students frequently fail to fix bugs by themselves even with the given testcases. Another method of feedback is to offer the answer code. However, it is still troublesome since there are many possible ways to implement a program for the specific problem; the given answers cannot help students to understand their mistakes in this case. Hence, providing failing testcases and answers is not sufficient for students who need more guided feedback.

Goal. The goal of this paper is to develop an automated system, called FixML, for generating personalized feedback on logical errors in functional programming assignments. Given a student's program that has logical errors as well as input-output testcases and a correct implementation from an instructor, FIxML automatically repairs the student's program by correcting buggy parts. The error location and an appropriate correction are presented to students, assisting them to better identify and understand their mistakes. Our target language is OCaml and we aim to provide feedback on not only introductory but also more challenging programming exercises.

Approach. To achieve this goal, we present a new error-correction algorithm for functional language. The algorithm combines statistical error-localization and enhanced type-directed program synthesis. First, it identifies the error locations and scores them with statistical reasoning on the results from dynamic executions. Secondly, the algorithm uses search-based program synthesis to replace the erroneous expression by a correct one that satisfies all of the given testcases. However, there remains a major scalability challenge for generating feedback on complex programs. Since we target a realistic subset of OCaml to deal with various types of problems, the search space for synthesis explodes. We address this challenge with an enhanced type-directed search algorithm. Due to the scalability issue, prior work on functional program synthesis [Feser et al. 2015; Frankle et al. 2016; Osera and Zdancewic 2015; Polikarpova et al. 2016] use type-directed search techniques. Although the technique can reduce the search space substantially, type-directed search alone was still unscalable to repair nontrivial student submissions. Our algorithm enhances type-directed enumerative search with two techniques: components reduction and search space pruning. Because enumerating all components is too large to search entirely, we reduce the components by deducing semantically redundant variables via data-flow analysis and using syntax components extracted from a correct implementation provided by instructor. To prune the search space more effectively, we combine type-directed enumeration with symbolic execution to detect the partial programs that are well-typed but functionally inconsistent. We formalize our algorithm for a subset of OCaml but the general idea is applicable to other functional languages as well.

Results. Our experimental results demonstrate that our approach effectively provides personalized feedback on variety of programming exercises. We evaluated our tool, FixML, with 497 students' programs from 13 problems with different levels. Overall, FixML successfully repaired $43 \%$ of the submissions in 5.4 seconds on average. Most notably, FIxML was able to accurately identify and fix an error in a large submission (154 lines, Appendix C). Furthermore, we show that FixML is actually helpful for students by conducting user study with 18 undergraduate students.

Proc. ACM Program. Lang., Vol. 2, No. OOPSLA, Article 158. Publication date: November 2018. 
Contributions. This paper makes the following contributions:

- We present FIxML, the first system for automatically diagnosing and correcting logical errors in functional programming exercises. While related existing work on functional languages has mostly focused on type errors (e.g. [Seidel et al. 2017; Wu et al. 2017; Wu and Chen 2017; Zhang et al. 2017]), our system aims to provide personalized feedback on logical errors.

- We present a novel error-correction algorithm that combines statistical error-localization and enhanced type-directed enumerative synthesis. We show that combining these techniques is a key to providing useful feedback on a wide range of nontrivial programs.

- We provide extensive evaluation results with real student submissions and user study. Our implementation and 497 benchmark programs are publicly available. ${ }^{1}$

\section{OVERVIEW}

\subsection{Motivating Examples}

Let us demonstrate FixML with three functional programming problems from an undergraduate Programming Languages course and StackOverflow. According to our experience, most students had difficulty in solving these problems on their own, often implemented erroneous programs, and required helps from human instructors to correct the errors.

As inputs, FIxML takes three components (Fig. 4): a student's program that has a logical error, a set of input-output testcases, which exposes the error, and a correct implementation. We assume that the last two components are provided from an instructor. Then, FIxML generates a program that passes all the testcases, by automatically fixing erroneous parts of the student's program.

Example 1 (Differentiation). Consider the problem of implementing a function that symbolically differentiates algebraic expressions. The expressions are defined in OCaml datatype as follows:

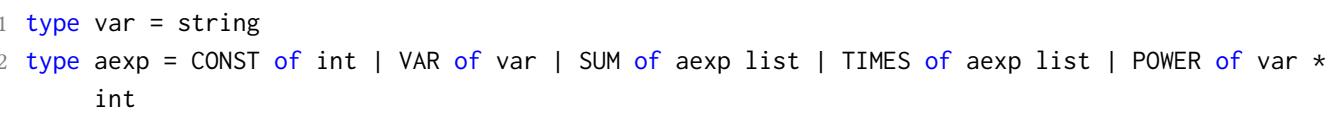

The goal of the problem is to define the function diff: aexp $*$ var $\rightarrow$ aexp, which takes an expression and a variable name as input and produces another expression obtained by differentiating the input expression with respect to the given variable. For example, the expression $x^{2}+1$ is represented by SUM [POWER (" $x ", 2$ ); CONST 1] and differentiating it with respect to $x$ gives $2 x$ which can be represented by TIMES [CONST 2; VAR " $x "$ ]. The main objective of this problem is to familiarize students with the use of inductive datatypes and recursive functions, both of which are essential in ML-style functional programming.

A student submitted an incorrect implementation shown in Fig. 1. The major part of the program is correct; for example, the student properly handles both the base and inductive cases for TIMES and SUM. However, the implementation has an error at line 10; when the base variable y is not equal to the variable $x$ given as argument, the result should be $\operatorname{CONST} 0$, not $\operatorname{POWER}(y, n)$ (e.g. differentiating $y^{2}$ with respect to $x$ produces 0 ).

FIXML is able to automatically generate the feedback in 1 second. Given the incorrect submission in Fig. 1, a correct implementation by an instructor, and a set of test-cases that expose the error of the submission, FIXML pinpoints the erroneous part (i.e. POWER $(y, n)$ at line 10) and replaces the expression by CONST 0 . In Fig. 1, the generated feedback is annotated as a comment at line 10 . The instructor's solution for this problem is presented in Appendix A.

FixML can correct diverse types of logical errors. For example, consider the incorrect program written by another student:

\footnotetext{
${ }^{1}$ https://github.com/kupl/FixML
} 


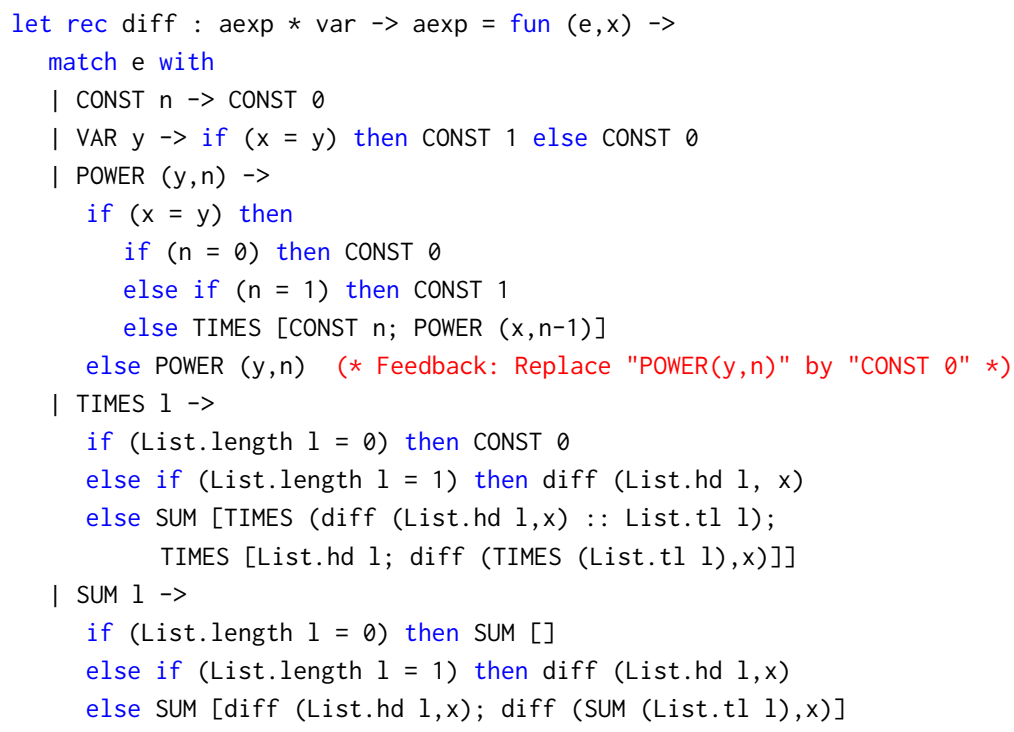

Fig. 1. Incorrect implementation for Example 1 (Differentiation)

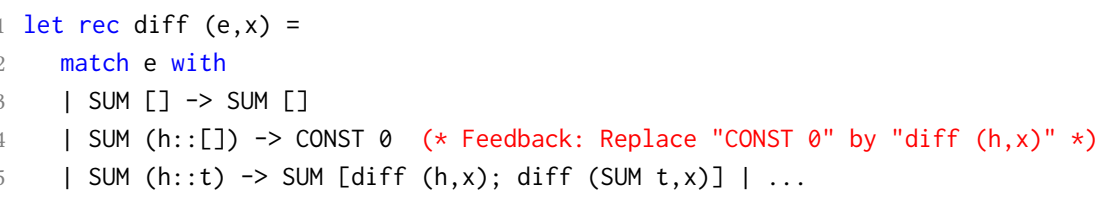

In this case, the program is incorrect as it does not differentiate the head element $h$ at line 4 . With the same solution and testcases, FIxML corrects the error by replacing the expression CONST $\theta$ by diff $(h, x)$ in 0.7 seconds.

Note that the two student submissions are substantially different from the instructor's solution. For example, in Appendix A, the instructor implemented the SUM case using List.map as follows (Problem \#13):

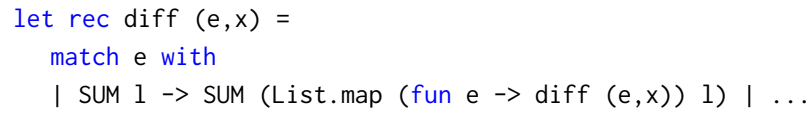

We observed that, for nontrivial programming assignments, students use many different ways of implementing the required functionality (see Section 5.5). The development of FIxML was motivated by the difference between the solution and submissions, which makes it difficult for students to identify and correct the errors in their own programs.

Furthermore, FIxML can introduce more complex expressions such as conditional expressions. For example, consider the following code:

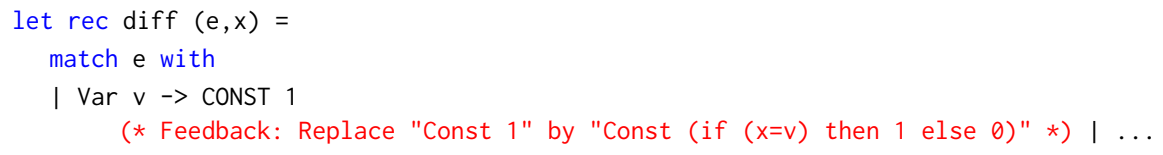




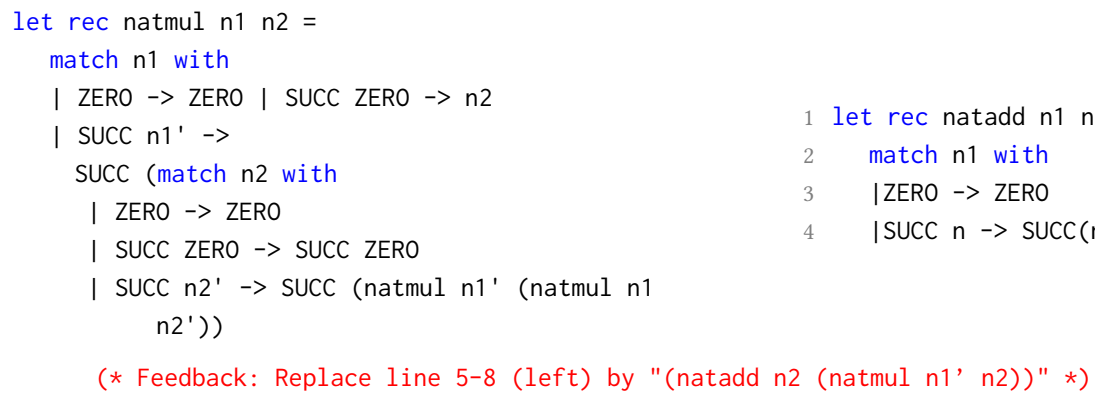

Fig. 2. Incorrect implementation for Example 2 (Natural Numbers)

The program has an error at line 3, where the student missed the case when the variable $v$ equals to the input variable $x$. FixML correctly identified this error and corrected the expression 1 by if var=str then 1 else 0 in 2.1 seconds.

Example 2 (Natural Numbers). The next problem is to implement functions that add and multiply user-defined natural numbers. The natural number can be defined in datatype as follows:

type nat $=$ ZERO | SUCC of nat

For instance, (SUCC (SUCC ZERO)) denotes 2. The goal of the problem is to define two functions natadd: nat $\rightarrow$ nat $\rightarrow$ nat and natmul: nat $\rightarrow>$ nat $\rightarrow$ nat, which take two natural numbers as input and produce their addition and multiplication, respectively. For example, natadd (SUCC (SUCC ZERO)) (SUCC ZERO) and natmul (SUCC (SUCC ZERO)) (SUCC ZERO) should produce (SUCC (SUCC (SUCC ZERO))) and (SUCC (SUCC ZERO))), respectively.

Fig. 2 shows an erroneous program written by a student, where natadd is correct but natmul has a big conceptual error. Note that multiplication is inductively defined with addition as follows:

$$
n_{1} \times n_{2}= \begin{cases}0 & n_{1}=0 \\ n_{2}+\left(n_{1}-1\right) \times n_{2} & n_{1} \neq 0\end{cases}
$$

However, the student could not conceive this equation and implemented the wrong codes at lines 58 , where a substantial modification is needed to correct the program. Impressively, FixML replaced the four lines (5-8) by the expression (natadd n2 (natmul n1' n2)) in 22 seconds.

Example 3 (Append). The last problem came from StackOverflow ${ }^{2}$, where the goal is to write append: 'a list ->'a list -> 'a list

which appends the first list to the second list while removing duplicated elements. For instance, given two lists $[4 ; 5 ; 6 ; 7]$ and $[1 ; 2 ; 3 ; 4]$, the function should output $[1 ; 2 ; 3 ; 4 ; 5 ; 6 ; 7]$ :

$$
\text { append }[4 ; 5 ; 6 ; 7][1 ; 2 ; 3 ; 4]=[1 ; 2 ; 3 ; 4 ; 5 ; 6 ; 7] \text {. }
$$

To solve the problem, one student defined the function append in Fig. 3. She first defined two helper functions: find and help_append. The function find takes an element and a list, and returns true iff the list contains the element. The purpose of the function help_append is to append elements of the list 11 to the list 12 if they are not in 12. Next, she defined the function append using help_append_list. Note that the implementation works correctly if the list 12 has no duplicated elements; for example, it produces the correct result for the example lists above. However, it does

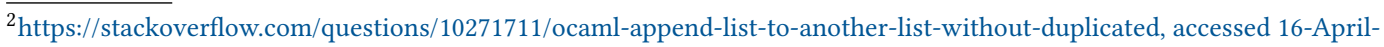
2018
} 


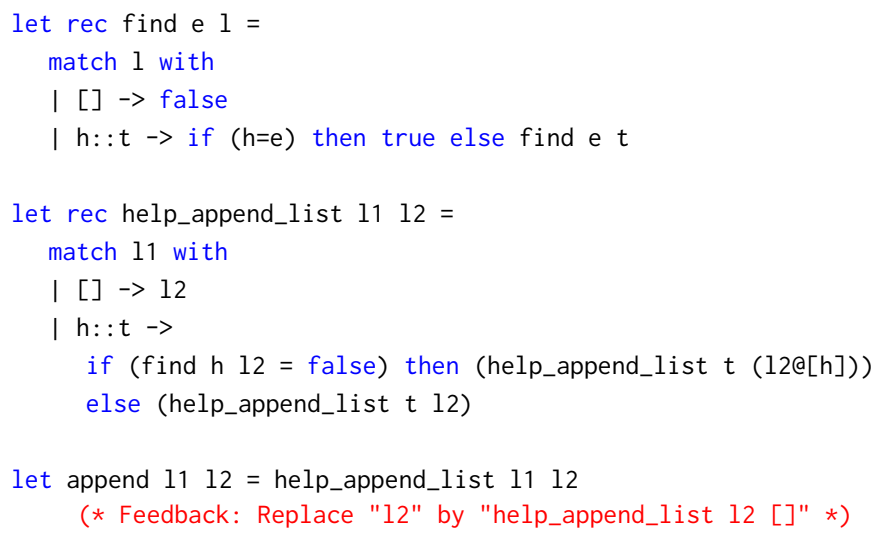

Fig. 3. Incorrection implementation for Example 3 (Append)

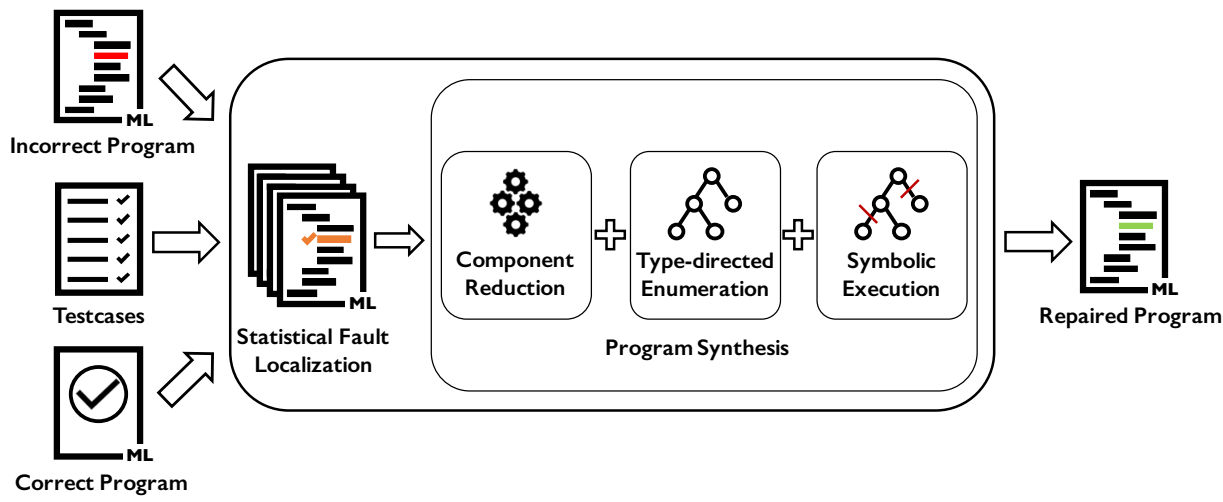

Fig. 4. Overview of FIXML.

not work when the members of 12 are redundant. For example, given the lists $[4 ; 5 ; 6 ; 7]$ and $[1 ; 2 ; 4 ; 4]$, the program outputs $[1 ; 2 ; 4 ; 4 ; 5 ; 6 ; 7]$, where 4 is duplicated. The student observed this faulty behavior but failed to diagnose and fix the error for herself.

FixML repairs the program in 43 seconds by removing duplicated elements in 12 before appending. The failure point of the original program is that it does not attempt to remove duplicates in 12 . FIXML precisely captures this root cause and modifies (help_append_list 11 12) at line 13 into (help_append_list 11 (help_append_list 12[] )), where duplicates in 12 are removed by applying help_append_list to 12 and [] (help_append_list correctly checks duplicates in the first argument list). We remark that this feedback is exactly the same as the manual feedback provided by a human in the post and agreed upon by several others without any objections.

\subsection{How FixML Works}

Fig. 4 illustrates how FixML works. Given a student's incorrect program, a set of testcases, and a correct implementation from an instructor, FIxML repairs the student code by using statistical error localization and enhanced type-directed synthesis. Let us illustrate our algorithm using a simple 


$$
\begin{aligned}
E::= & U^{l}, \quad p::=c\left(x_{1}, \ldots, x_{k}\right), \quad \tau::=T\left|\tau_{1} \rightarrow \tau_{2}\right| \alpha \\
U::= & x|\lambda x . E| E_{1} E_{2} \mid \text { let } x=E_{1} \text { in } E_{2} \mid \text { letrec } f(x)=E_{1} \text { in } E_{2} \mid c\left(E_{1}, \ldots, E_{k}\right) \\
& \mid \text { match } E_{0} \text { with } \overline{p_{i} \rightarrow E_{i}} k \mid \square
\end{aligned}
$$

Fig. 5. Syntax of simplified language

given testcase, we conclude that the partial program cannot be a solution. For example, suppose a testcase $\{3 \mapsto 6\}$ and a partial program (let factorial $\mathrm{n}=$ match $\mathrm{n}$ with $|0 \rightarrow 1| \mathrm{x} \rightarrow$ $x \star x *$ ?) are given. Continuing search starting from the partial program would eventually fail, as the symbolic execution produces the unsatisfiable constraint $3 * 3 * ?=6$.

\section{PROBLEM DEFINITION}

Language. FixML targets a significant subset of OCaml. In particular, it has been designed to handle a variety of functional programming exercises including not only introductory but also more advanced problems. For example, FIxML is expressive enough to cover all programming exercises used in a senior-level Programming Languages course taught by the authors of this paper. The full language is given in Appendix B but, for brevity, we illustrate our approach with a small language in Fig. 5, where $E$ and $U$ denote labelled and unlabelled expressions. Expressions include variable $(x)$, function definition $(\lambda x . E)$, function application $\left(E_{1} E_{2}\right)$, let-expression (let $x=E_{1}$ in $\left.E_{2}\right)$, recursive function definition (letrec $f(x)=E_{1}$ in $\left.E_{2}\right)$, user-defined type constructor $\left(c\left(E_{1}, \ldots, E_{k}\right)\right.$, where $c$ is the constructor name, and pattern-matching (match $E$ with ${\overline{p_{i}} \rightarrow E_{i}}^{k}$, where ${\overline{p_{i} \rightarrow E_{i}}}^{k}$ denotes $p_{1} \rightarrow E_{1}|\cdots| p_{k} \rightarrow E_{k}$ ). An exceptional syntactic element is a hole ( $\square$ ), which is a placeholder that will get replaced by a complete expression by our algorithm. Initial programs do not contain holes. A pattern $(p)$ is a user-defined type constructor with variable arguments $\left(c\left(x_{1}, \ldots, x_{k}\right)\right)$. Types $(\tau)$ include user-defined algebraic data types $(T)$, function types $\left(\tau_{1} \rightarrow \tau_{2}\right)$, and type variable $(\alpha)$. In the rest of this paper, we write $I d$ for the set of identifiers, $L a b$ for the set of labels, and $\Lambda$ for a mapping from constructor names to their types declared in the program (i.e. $\left.\Lambda=\left[c_{1} \mapsto\left(\tau_{1} * \cdots * \tau_{k}\right) \rightarrow T_{1}, \ldots\right]\right)$.

Let $\mathcal{E} \llbracket E \rrbracket: E n v \rightarrow$ Val be the evaluation function, where an environment $\rho \in E n v=I d \rightarrow V a l$ maps variables $(I d)$ to values ( Val) and values are user-defined values (Cnstr), functions (Closure), and recursive functions (RecClosure): Val $=$ Cnstr + Closure + RecClosure, where Cnstr $=I d \times V a l^{*}$ (constructor name and values), Closure $=I d \times E \times E n v$ (argument, body, and environment), and RecClosure $=I d \times I d \times E \times E n v$ (function name, argument, body, and environment). Semantics is standard and defined only for expressions without holes.

The Correction Problem. Our error-correction problem is defined with the four components:

$$
\left(x, E, E_{c}, \mathcal{T}\right)
$$

where $x$ is an input variable, $E$ is a program written by a student, $E_{c}$ is a correct implementation provided by an instructor, and $\mathcal{T} \in \mathrm{Val} \times \mathrm{Val}$ is a set of input-output testcases. We assume that functions are not the final value, $x$ is the only variable that appears free in $E$ and $E_{c}$ (i.e., $\left.F V(E)=F V\left(E_{c}\right)=\{x\}\right)$, and the program $E$ is incorrect with respect to the testcases:

$$
\exists\left(v_{i}, v_{o}\right) \in \mathcal{T} . \mathcal{E} \llbracket E \rrbracket\left(\left[x \mapsto v_{i}\right]\right) \neq v_{o} .
$$

Then, our goal is to find a program $E^{\prime}$ that works correctly for all testcases:

$$
\forall\left(v_{i}, v_{o}\right) \in \mathcal{T} . \mathcal{E} \llbracket E^{\prime} \rrbracket\left(\left[x \mapsto v_{i}\right]\right)=v_{o} .
$$


Note that our aim is not to simply return the correct implementation $E_{c}$ but instead generates $E^{\prime}$ from $E$. Our algorithm works even without $E_{c}$, but we require it for extracting common syntax components (Section 4.3), which increases the efficiency of our algorithm.

\section{ERROR CORRECTION ALGORITHM}

In this section, we describe our error-correction algorithm (Algorithm 1). Section 4.1 explains the error localization procedure, which identifies a set of suspicious expressions that may contain errors. Our algorithm attempts to replace each of these expressions by correct one until it finds a program that passes all testcases. In Sections 4.2, 4.3, and 4.4, we formalize this correction process as a state-search procedure that is able to find a correction efficiently by combining type-directed enumeration, components reduction, and pruning by symbolic execution.

\subsection{Statistical Error Localization}

The algorithm begins with localizing errors. To locate errors in a program, we adapt a well-known statistical technique [Jones et al. 2002] for functional programs. Given a program $E$ which is buggy with respect to testcases $\mathcal{T}$, our technique produces a set of pairs, where each pair $\left(E_{\square}, s\right)$ consists of a holed program $E_{\square}$ and its score $s . E_{\square}$ is obtained from $E$ by substituting a suspicious subexpression of $E$ with a hole ( $\square$ ). The score $s$ measures how suspicious the substituted expression is.

The localization procedure takes the input variable $x$, buggy program $E$, and testcases $\mathcal{T}$. It first classifies the testcases into positive $(P)$ and negative $(N)$ testcases. We say a testcase $\left(v_{i}, v_{o}\right) \in \mathcal{T}$ is positive if the program $E$ with $v_{i}$ correctly evaluates to $v_{o}$. Otherwise, the testcase is called negative. The sets $P$ and $N$ are defined as follows:

$$
P=\left\{\left(v_{i}, v_{o}\right) \in \mathcal{T} \mid \mathcal{E} \llbracket E \rrbracket\left[x \mapsto v_{i}\right]=v_{o}\right\}, \quad N=\left\{\left(v_{i}, v_{o}\right) \in \mathcal{T} \mid \mathcal{E} \llbracket E \rrbracket\left[x \mapsto v_{i}\right] \neq v_{o}\right\} .
$$

Next, we collect subexpressions of $E$ that are evaluated under the program execution with negative testcases. Let $S$ be the set of all subexpressions of the program $E$. We assume a predicate $V: S \times \mathcal{T} \rightarrow$ $\{0,1\}$ such that, given a subexpression $e^{l} \in S$ and a testcase $t \in \mathcal{T}, V\left(e^{l}, t\right)$ is 1 iff the expression $e^{l}$ is evaluated when $E$ runs under the testcase $t$. Then, our localization procedure produces the following set of candidates for repair:

$$
C=\left\{\left(e^{l}, \operatorname{hole}(E, l), \operatorname{score}\left(e^{l}\right)\right) \mid e^{l} \in S \wedge \exists t \in N . V\left(e^{l}, t\right)=1\right\} .
$$

A candidate $\left(e, E_{\square}, s\right) \in C$ includes a potentially erroneous subexpression $e$ of $E$, a holed expression $E_{\square}$, and the score of $e$. The function hole $(E, l)$ replaces the subexpression $e$, whose label is $l$, of $E$ by a hole, which is inductively defined in a straightforward way: e.g.,

$$
\operatorname{hole}\left(x^{l}, l^{\prime}\right)=\left\{\begin{array}{ll}
\square^{l} & \text { if } l=l^{\prime} \\
x^{l} & \text { if } l \neq l^{\prime}
\end{array} \quad \operatorname{hole}\left((\lambda x . E)^{l}, l^{\prime}\right)= \begin{cases}\square^{l} & \text { if } l=l^{\prime} \\
\left(\lambda x \cdot \operatorname{hole}\left(E, l^{\prime}\right)\right)^{l} & \text { if } l \neq l^{\prime}\end{cases}\right.
$$

We compute the score of suspicious subexpression $e$ as follows (lower is more suspicious):

$$
\operatorname{score}(e)=\operatorname{size}(e)+\frac{\sum_{p \in P} V(e, p)}{\sum_{t \in T} V(e, t)} \times \frac{\sum_{\left(e_{,-,}\right) \in C} \operatorname{size}\left(e^{\prime}\right)}{|C|}
$$

where size $(e)$ denotes the size of $e$, which is heuristically estimated based on Occam's razor. The idea is that we would like to repair as small a subexpression as possible $(\operatorname{size}(e))$ and we presume that subexpressions that are less involved in positive test runs are more likely to be erroneous $\left(\frac{\sum_{p \in P} V(e, p)}{\sum_{t \in T} V(e, t)}\right)$. The last term $\left(\frac{\sum_{\left(e^{\prime}, \ldots\right) \in C} \operatorname{size}\left(e^{\prime}\right)}{|C|}\right)$ is the normalization factor that scales the second term in accordance with the first term. 


$$
\begin{aligned}
& \frac{x \in \Sigma(l) \quad S=\operatorname{unify}(\Upsilon(l), \Gamma(l)(x))}{\left\langle\square^{l}, \Upsilon, \Gamma\right\rangle \rightsquigarrow \Sigma, \Omega\left\langle x^{l}, S(\Upsilon), S(\Gamma)\right\rangle} \\
& \lambda x . \square \in \Omega \quad \Upsilon\left(l_{1}\right)=\tau_{1} \rightarrow \tau_{2} \quad \text { new } l_{2} \\
& \overline{\left\langle\square^{l_{1}}, \Upsilon, \Gamma\right\rangle \rightsquigarrow_{\Sigma, \Omega}\left\langle\left(\lambda x . \square^{l_{2}}\right)^{l_{1}}, \Upsilon\left[l_{2} \mapsto \tau_{2}\right], \Gamma\left[l_{2} \mapsto\left(\Gamma\left(l_{1}\right)\right)\left[x \mapsto \tau_{1}\right]\right]\right\rangle}
\end{aligned}
$$

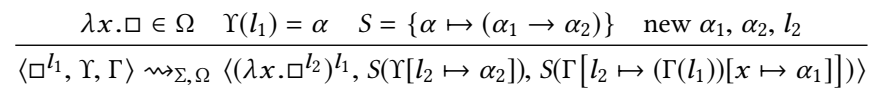

$$
\begin{aligned}
& (\square \square) \in \Omega \quad \Upsilon(l)=\tau \quad \text { new } \alpha, l_{1}, l_{2}
\end{aligned}
$$

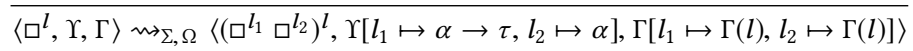

$$
\begin{aligned}
& \text { (let } x=\square \text { in } \square) \in \Omega \quad \Upsilon(\square)=\tau \quad \text { new } \alpha, l_{1}, l_{2}
\end{aligned}
$$

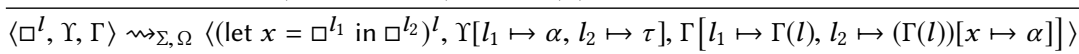

$$
\begin{aligned}
& \text { (letrec } f(x)=\square \text { in } \square) \in \Omega \quad \Upsilon(l)=\tau \quad \text { new } \alpha_{1}, \alpha_{2}, l_{1}, l_{2} \\
& \left\langle\square^{l}, \Upsilon, \Gamma\right\rangle \rightsquigarrow_{\Sigma, \Omega}\left\langle\left(\text { letrec } f(x)=\square^{l_{1}} \text { in } \square^{l_{2}}\right)^{l}, \Upsilon\left[\begin{array}{l}
l_{1} \mapsto \alpha_{1} \\
l_{2} \mapsto \tau
\end{array}\right], \Gamma\left[\begin{array}{l}
l_{1} \mapsto(\Gamma(l))\left[f \mapsto\left(\alpha_{1} \rightarrow \alpha_{2}\right), x \mapsto \alpha_{1}\right] \\
l_{2} \mapsto(\Gamma(l))\left[f \mapsto\left(\alpha_{1} \rightarrow \alpha_{2}\right)\right]
\end{array}\right]\right\rangle \\
& \frac{\Lambda(c)=\left(\tau_{1} * \cdots * \tau_{k} \rightarrow T\right) \quad \Upsilon(l)=T \quad \text { new } l_{1}, \ldots, l_{k}}{\left\langle\square^{l}, \Upsilon, \Gamma\right\rangle \rightsquigarrow \Sigma, \Omega\left\langle\left(c\left(\square^{l_{1}}, \ldots, \square^{l_{k}}\right)\right)^{l}, \Upsilon\left[l_{i} \mapsto \tau_{i}\right]_{i=1}^{k}, \Gamma\left[l_{i} \mapsto \Gamma(l)\right]_{i=1}^{k}\right\rangle} \\
& \frac{\Lambda(c)=\left(\tau_{1} * \cdots * \tau_{k} \rightarrow T\right) \quad \Upsilon(l)=\alpha \wedge S=\{\alpha \mapsto T\} \quad \text { new } l_{1}, \ldots, l_{k}}{\left\langle\square^{l}, \Upsilon, \Gamma\right\rangle \rightsquigarrow \Sigma, \Omega\left\langle\left(c\left(\square^{l_{1}}, \ldots, \square^{l_{k}}\right)\right)^{l}, S\left(\Upsilon\left[l_{i} \mapsto \tau_{i}\right]_{i=1}^{k}\right), S\left(\Gamma\left[l_{i} \mapsto \Gamma(l)\right]_{i=1}^{k}\right)\right\rangle} \\
& \Upsilon(\square)=\tau \quad \text { typeof }\left(p_{i}, \Lambda\right)=\tau_{p} \\
& \left\langle\square^{l}, \Upsilon, \Gamma\right\rangle \rightsquigarrow \Sigma, \Omega\left\langle\left(\text { match } \square^{l_{0}} \text { with } \overline{p_{i} \rightarrow \square^{l_{i}}}\right)^{k},\left(\Upsilon\left[l_{0} \mapsto \tau_{p}\right]\right)\left[l_{i} \mapsto \tau\right]_{i=1}^{k},\left(\Gamma\left[l_{0} \mapsto \Gamma(l)\right]\right)\left[l_{i} \mapsto \operatorname{bindtype}\left(p_{i}, \Lambda\right)\right]_{i=1}^{k}\right\rangle \\
& \frac{\langle E, \Upsilon, \Gamma\rangle \rightsquigarrow_{\Sigma, \Omega}\left\langle E^{\prime}, \Upsilon^{\prime}, \Gamma^{\prime}\right\rangle}{\langle x . E, \Upsilon, \Gamma\rangle \rightsquigarrow_{\Sigma, \Omega}\left\langle\lambda x . E^{\prime}, \Upsilon^{\prime}, \Gamma^{\prime}\right\rangle} \frac{\left\langle E_{1}, \Upsilon, \Gamma\right\rangle \rightsquigarrow_{\Sigma, \Omega}\left\langle E_{1}^{\prime}, \Upsilon^{\prime}, \Gamma^{\prime}\right\rangle}{\left\langle E_{1} E_{2}, \Upsilon, \Gamma\right\rangle \rightsquigarrow_{\Sigma, \Omega}\left\langle E_{1}^{\prime} E_{2}, \Upsilon^{\prime}, \Gamma^{\prime}\right\rangle} \frac{\left\langle E_{2}, \Upsilon, \Gamma\right\rangle \rightsquigarrow_{\Sigma, \Omega}\left\langle E_{2}^{\prime}, \Upsilon^{\prime}, \Gamma^{\prime}\right\rangle}{\left\langle E_{1} E_{2}, \Upsilon, \Gamma\right\rangle \rightsquigarrow_{\Sigma, \Omega}\left\langle E_{1} E_{2}^{\prime}, \Upsilon^{\prime}, \Gamma^{\prime}\right\rangle} \\
& \left\langle E_{1}, \Upsilon, \Gamma\right\rangle \rightsquigarrow_{\Sigma, \Omega}\left\langle E_{1}^{\prime}, \Upsilon^{\prime}, \Gamma^{\prime}\right\rangle \\
& \overline{\left\langle\text { let } x=E_{1} \text { in } E_{2}, \Upsilon, \Gamma\right\rangle \rightsquigarrow_{\Sigma, \Omega}\left\langle\text { let } x=E_{1}^{\prime} \text { in } E_{2}, \Upsilon^{\prime}, \Gamma^{\prime}\right\rangle} \overline{\left\langle\text { let } x=E_{1} \text { in } E_{2}, \Upsilon, \Gamma\right\rangle \rightsquigarrow_{\Sigma, \Omega}\left\langle\text { let } x=E_{1} \text { in } E_{2}^{\prime}, \Upsilon^{\prime}, \Gamma^{\prime}\right\rangle}
\end{aligned}
$$

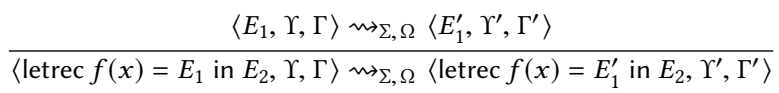

$$
\begin{aligned}
& \left\langle E_{2}, \Upsilon, \Gamma\right\rangle \rightsquigarrow \Sigma, \Omega\left\langle E_{2}^{\prime}, \Upsilon^{\prime}, \Gamma^{\prime}\right\rangle \\
& \overline{\left\langle\text { letrec } f(x)=E_{1} \text { in } E_{2}, \Upsilon, \Gamma\right\rangle \leadsto \Sigma, \Omega}\left\langle\text { letrec } f(x)=E_{1} \text { in } E_{2}^{\prime}, \Upsilon^{\prime}, \Gamma^{\prime}\right\rangle \\
& \frac{\left\langle E_{1}, \Upsilon, \Gamma\right\rangle \rightsquigarrow \Sigma, \Omega}{\frac{\left.E_{1}^{\prime}, \Upsilon^{\prime}, \Gamma^{\prime}\right\rangle}{\left\langle c\left(E_{1}, \ldots, E_{k}\right), \Upsilon, \Gamma\right\rangle \rightsquigarrow \Sigma, \Omega}\left\langle c\left(E_{1}^{\prime}, \ldots, E_{k}\right), \Upsilon^{\prime}, \Gamma^{\prime}\right\rangle} \quad \frac{\left\langle E_{k}, \Upsilon, \Gamma\right\rangle \rightsquigarrow_{\Sigma, \Omega}\left\langle E_{k}^{\prime}, \Upsilon^{\prime}, \Gamma^{\prime}\right\rangle}{\left\langle c\left(E_{1}, \ldots, E_{k}\right), \Upsilon, \Gamma\right\rangle \aleph_{\Sigma, \Omega}\left\langle c\left(E_{1}, \ldots, E_{k}^{\prime}\right), \Upsilon^{\prime}, \Gamma^{\prime}\right\rangle} \\
& \langle E, \Upsilon, \Gamma\rangle \rightsquigarrow_{\Sigma, \Omega}\left\langle E^{\prime}, \Upsilon^{\prime}, \Gamma^{\prime}\right\rangle \\
& \overline{\left\langle\text { match } E \text { with } p_{1} \rightarrow E_{1}|\cdots| p_{k} \rightarrow E_{k}, \Upsilon, \Gamma\right\rangle \rightsquigarrow \aleph_{, \Omega}\left\langle\text { match } E^{\prime} \text { with } p_{1} \rightarrow E_{1}|\cdots| p_{k} \rightarrow E_{k}, \Upsilon^{\prime}, \Gamma^{\prime}\right\rangle} \\
& \left\langle E_{k}, \Upsilon, \Gamma\right\rangle m_{\Sigma, \Omega}\left\langle E_{k}^{\prime}, \Upsilon^{\prime}, \Gamma^{\prime}\right\rangle \\
& \overline{\left\langle\text { match } E \text { with } p_{1} \rightarrow E_{1}|\cdots| p_{k} \rightarrow E_{k}, \Upsilon, \Gamma\right\rangle \rightsquigarrow_{\Sigma, \Omega}\left\langle\text { match } E \text { with } p_{1} \rightarrow E_{1}^{\prime}|\cdots| p_{k} \rightarrow E_{k}, \Upsilon^{\prime}, \Gamma^{\prime}\right\rangle} \\
& \left\langle E_{1}, \Upsilon, \Gamma\right\rangle \rightsquigarrow \rightsquigarrow_{\Sigma, \Omega}\left\langle E_{1}^{\prime}, \Upsilon^{\prime}, \Gamma^{\prime}\right\rangle \\
& \overline{\left\langle\text { match } E \text { with } p_{1} \rightarrow E_{1}|\cdots| p_{k} \rightarrow E_{k}, \Upsilon, \Gamma\right\rangle \rightsquigarrow \Sigma, \Omega}\left\langle\text { match } E \text { with } p_{1} \rightarrow E_{1}^{\prime}|\cdots| p_{k} \rightarrow E_{k}, \Upsilon^{\prime}, \Gamma^{\prime}\right\rangle
\end{aligned}
$$

Fig. 6. Type-directed transition relation. 


\subsection{Type-Directed Enumerative Search}

After locating errors, the algorithm attempts to fix them by synthesizing correct expressions for holes in partial programs. To do so, it basically performs enumerative search, exhaustively generating all the possible expressions according to the language grammar in increasing size. However, this simple approach does not work as our language (Appendix B) is too large. To make it practical, we use three techniques: type-directed enumeration, components reduction, and pruning via symbolic execution.

We first describe type-directed enumerative search, which is defined by the transition system:

$$
(S, \rightsquigarrow, I, F)
$$

where $S$ is a set of states, $(\rightsquigarrow) \subseteq S \times S$ is a transition relation, $I \subseteq S$ is a set of initial states, and $F \subseteq S$ is a set of final states.

States. A state $s \in S$ is a triple $(E, \Upsilon, \Gamma)$, where $E$ is a partial program that may have holes and $\Upsilon$ and $\Gamma$ are type environments for holes and variables. $\Upsilon:$ Hole $\rightarrow$ Type is a type environment that maps holes to their types. $\Gamma:$ Hole $\rightarrow(I d \rightarrow$ Type $)$ stores types of variables for each hole of the program. For example, when $E_{1}=\left(\right.$ let $x=1$ in $\left.x+\square^{l}\right), \Upsilon$ and $\Gamma$ store the following information: $\Upsilon=[l \mapsto$ int $], \Gamma=[l \mapsto[x \mapsto$ int $]]$. The type of hole $l$ is int and variable $x$ has type int at the hole $l$. An important role of $\Gamma$ is to keep track of variables that can appear free inside each hole. For example, when $E_{2}=\left(\right.$ let $x=\square^{l_{1}}$ in let $y=x$ in $\square^{l_{2}}$ ), $\Gamma$ stores the information: $\Gamma=\left[l_{1} \mapsto[], l_{2} \mapsto[x \mapsto \alpha, y \mapsto \alpha]\right]$, which indicates that no variables and variables $\{x, y\}$ can be used without definitions at the first and second holes, respectively. We write $\operatorname{dom}(\Gamma(l))$ for the set of variables available at the location $l$ : e.g., in $E_{2}, \operatorname{dom}\left(\Gamma\left(l_{1}\right)\right)=\emptyset$ and $\operatorname{dom}\left(\Gamma\left(l_{2}\right)\right)=\{x, y\}$. In our algorithm, maintaining these two type environments enables to perform an efficient type-directed search.

Initial and Final States. The set $I$ of initial states is defined as follows:

$$
I=\left\{\left(E_{0}, \Upsilon_{0}, \Gamma_{0}\right) \mid\left(, E_{0},{ }_{-}\right) \in C, \Upsilon_{0} \text { and } \Gamma_{0} \text { are initial type environments for } E_{0}\right\}
$$

where $C$ is the set of repair candidates in (1) that results from the localization procedure. $\Upsilon$ and $\Gamma$ can be easily obtained by running a standard type inference algorithm with a slight modification to assign a fresh type variable $(\alpha)$ to each hole in $E_{0}$. The goal of search is to find a final state $(E, \Upsilon, \Gamma)$ such that $E$ does not have any holes and is correct with respect to the given testcases: $F=\left\{\left(E,{ }_{-},{ }_{-}\right) \in S \mid \forall\left(v_{i}, v_{o}\right) \in \mathcal{T} \cdot \mathcal{E} \llbracket E \rrbracket\left(\left[x \mapsto v_{i}\right]\right)=v_{o}\right\}$.

Transition Relation. Given a state $s$, transition relation $\left(\rightsquigarrow_{\Sigma, \Omega}\right) \subseteq S \times S$ determines the next states of $s$. Fig. 6 defines the transition relation as a set of inference rules. In the definition, we assume the standard unification algorithm, unify $: \tau \times \tau \times$ Subst $\rightarrow$ Subst, and the subscripts $\Sigma$ and $\Omega$ represent the syntax and variable components that we are allowed to use during synthesis. For the moment, we assume that the algorithm simply uses all syntax components of the language:

$$
\Omega=\left\{\lambda x . \square, \square \square \text {, let } x=\square \text { in } \square \text {, letrec } f(x)=\square \text { in } \square \text {, match } \square \text { with } p_{1} \rightarrow \square|\cdots| p_{k} \rightarrow \square\right\}
$$

and all available variables, i.e., $\Sigma=\lambda l$. $\operatorname{dom}(\Gamma(l))$.

Note that state transitions between states occur in a type-directed manner; ill-typed partial programs never appear during the transitions. For example, the rule

$$
\frac{\lambda x . \square \in \Omega \quad \Upsilon\left(l_{1}\right)=\tau_{1} \rightarrow \tau_{2} \quad \text { new } l_{2}}{\left\langle\square^{l_{1}}, \Upsilon, \Gamma\right\rangle \rightsquigarrow_{\Sigma, \Omega}\left\langle\left(\lambda x . \square^{l_{2}}\right)^{l_{1}}, \Upsilon\left[l_{2} \mapsto \tau_{2}\right], \Gamma\left[l_{2} \mapsto\left(\Gamma\left(l_{1}\right)\right)\left[x \mapsto \tau_{1}\right]\right]\right\rangle}
$$

says that the hole $\square^{l_{1}}$ is able to get replaced by $\lambda x . \square^{l_{2}}$ only when the hole $\square^{l_{1}}$ is of a function type (i.e. $\Upsilon\left(l_{1}\right)=\tau_{1} \rightarrow \tau_{2}$ for some type $\tau_{1}$ and $\tau_{2}$ ). The type environments $\Upsilon$ and $\Gamma$ are updated 


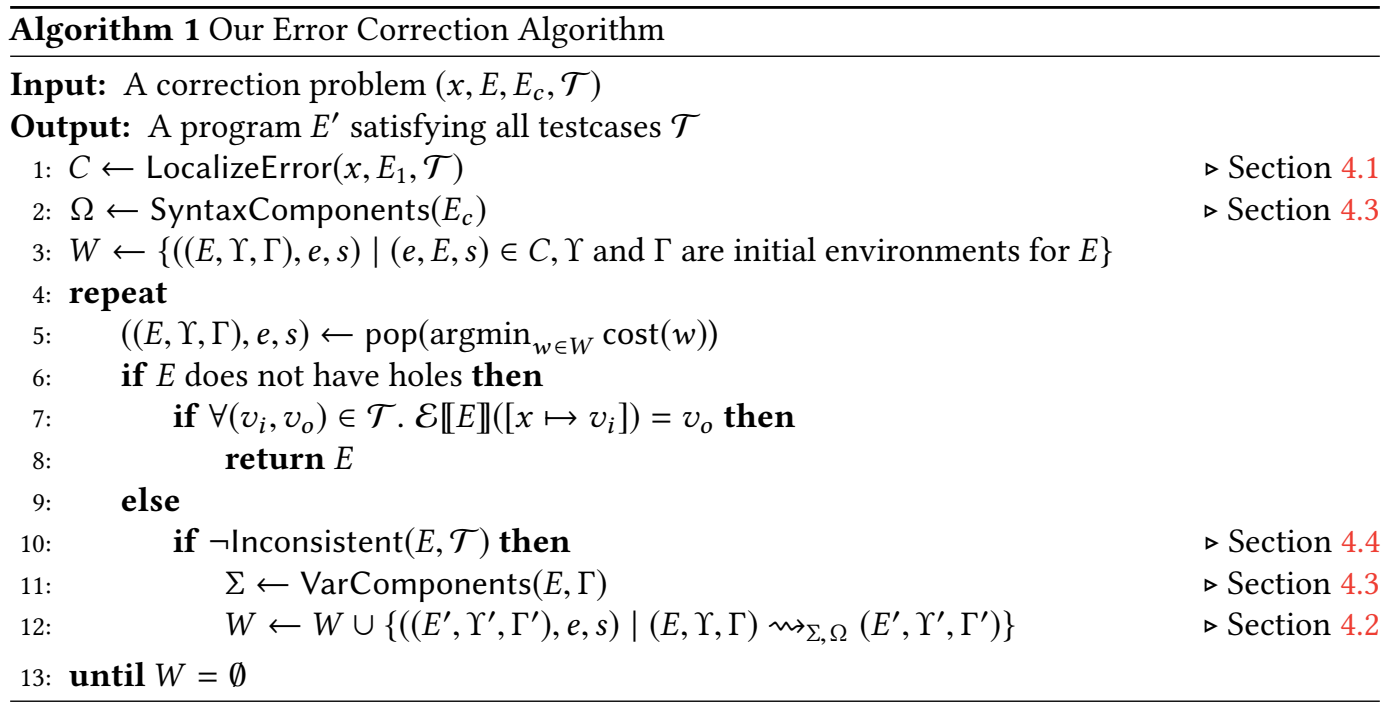

accordingly: $\Upsilon$ is updated so that the new hole $\square^{l_{2}}$ has type $\tau_{2}$ (i.e., $\Upsilon\left[l_{2} \mapsto \tau_{2}\right]$ ), and $\Gamma$ stores the variable-type information for the newly introduced hole i.e., $\Gamma\left[l_{2} \mapsto\left(\Gamma\left(l_{1}\right)\right)\left[x \mapsto \tau_{1}\right]\right]$.

Overall Algorithm. Lines 4-13 of Algorithm 1 describe our algorithm for type-directed enumerative search. It is a workset algorithm, where a workset element $w=((E, \Upsilon, \Gamma), e, s)$ consists of a state $(E, \Upsilon, \Gamma)$, the original expression $e$ for the hole, and the score of $e$. At line 5 , we choose a workset element $w$ with a lowest cost:

$$
\operatorname{cost}((E, \Upsilon, \Gamma), e, s)=\operatorname{size}(E)+s .
$$

Intuitively, we would like to produce a minimal correction $(\operatorname{size}(E))$ and make minimal changes from the original incorrect program ( $s$, the suspicious score obtained in Section 4.1). If the chosen program $E$ does not have any holes (line 6), we check whether $E$ is a final state (line 7). If a final state is found, it is returned and the algorithm terminates (line 8). Otherwise, we check if the current program $E$ is consistent with the given testcases (line 10). If so, we determine the variable components (line 12) and update $W$ so that it includes all the next states according to the transition relation. During the algorithm, we exclude potentially non-terminating programs using three heuristics. Firstly, we do not allow recursive calls in every branch of a program, e.g., (letrec $f x=$ match $x$ with $p_{1} \rightarrow f \square \mid p_{2} \rightarrow f \square$ ). Secondly, we do not allow recursive calls with unchanging arguments, e.g, (letrec $f x=$ match $x$ with $p_{1} \rightarrow f x \mid p_{2} \rightarrow \square$ ). Finally, when we evaluate the candidate program (line 7 ), we abort its execution if it runs exceeding a predetermined time limit.

\subsection{Reducing Program Components}

We further accelerate enumerative search by reducing the program components (i.e. $\Sigma$ and $\Omega$ in Fig. 6). In particular, we reduce the variable components $(\Sigma)$ by running a data-flow analysis that identifies semantically equivalent variables. To reduce the syntax components $(\Omega)$, we focus on the components used in the correct implementation $E_{c}$ provided by an instructor.

Motivation. One major factor that degrades the performance of enumerative search is the large set of program variables the algorithm is allowed to use during the synthesis process. Our 
algorithm does not use all available variables but carefully chooses a subset of them without losing expressiveness. Consider the following code snippet:

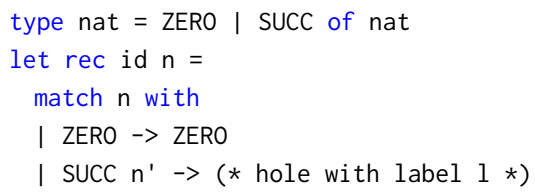

At line 5, although two variables $n$ and $n$ ' can be used by the synthesis procedure, we do not use $n$ because $n$ can be expressed in terms of $n$ ', i.e., $n=$ SUCC $n^{\prime}$ at the hole. This way, our algorithm avoids to enumerate all available variables, increasing the efficiency significantly, but doing so does not lose any chance of finding a solution.

Data-Flow Analysis. To do so, we designed a simple data-flow analysis that identifies semantically equivalent variables (or patterns). The goal of analysis is to produce the following map:

$$
M: L a b \rightarrow \wp((I d+P a t) \times(I d+P a t)) .
$$

Given a label $l \in L a b, M(l)$ stores the set of equivalent variables (or patterns) right before evaluating the $l$-labelled subexpression. For example, in the code snippet above, $M(l)=\left\{\left(\mathrm{n}\right.\right.$, SUCC $\left.\left.\mathrm{n}^{\prime}\right)\right\}$, meaning that $\mathrm{n}$ and SUCC $\mathrm{n}$ ' always have the same value right before the label $l$. Formally, we write $M \mid=E$ for the case that $M$ is correct with respect to the expression $E$. The correctness specification is given in Fig. 7. The specification can be easily translated to a top-down data-flow analysis algorithm. For example, when $E=\left(E_{1}^{l_{1}} E_{2}^{l_{2}}\right)^{l}$, we propagate the equivalence relation at $l$ to both $l_{1}$ (i.e. $\left.M(l) \subseteq M\left(l_{1}\right)\right)$ and $l_{2}$ (i.e. $M(l) \subseteq M\left(l_{2}\right)$ ). Facts are killed and generated at the function definitions, let-binding, and match. For example, consider $E=\left(\text { let } x=E_{1}^{l_{1}} \text { in } E_{2}^{l_{2}}\right)^{l}$. Starting from the facts $M(l)$ at $l$, we update the relationships by removing old facts $(\operatorname{kill}(M(l),\{x\}))$, generating new facts $\left(\operatorname{gen}\left(x, E_{1}\right)\right)$, and closuring the result. The gen and kill functions are responsible for generating and removing facts according to the semantics of expressions.

Using the Analysis Result. With the analysis, we refine the variable components $(\Sigma)$ as follows. Given the current partial program $E$ during the search procedure (line 11 in Algorithm 1), we analyze the program and obtain the information $M_{E}$ about equivalent variables. With $M_{E}$, our algorithm uses the following sets of variables for each label $l$ :

$$
\operatorname{VarComponents}(E, \Gamma)=\lambda l \cdot \operatorname{dom}(\Gamma(l)) \backslash\left\{x \in I d \mid\left(\_, x\right) \in M_{E}(l)\right\} .
$$

That is, we use variables if they do not appear in constructors (i.e. $x \in I d$ ) and they can be replaced by other variables (i.e. $\left.(\ldots, x) \in M_{E}(l)\right)$. Let us justify the second condition. For each pair $\left(v_{1}, v_{2}\right) \in M(l)$, we have the three possible cases:

- When $\left(v_{1}, v_{2}\right)=(y, x)$ : It is fine to exclude the variable $x$, because we can express $x$ by a more recently defined variable $y$.

- When $\left(v_{1}, v_{2}\right)=\left(c\left(x_{1}, \ldots, x_{k}\right), x\right)$ : It is also fine to exclude the variable $x$, because we can express $x$ using the variables $x_{1}, \ldots, x_{k}$ with the constructor $c$.

- When $\left(v_{1}, v_{2}\right)=\left(x, c\left(x_{1}, \ldots, x_{k}\right)\right)$ : We should not remove the variables $x_{1}, \ldots, x_{k}$, because they cannot be expressed by $x$. Instead, we can remove $x$, as we do in the second case. However, we also use $x$ during the synthesis process, considering the readability of synthesized results (more recently defined variables are more likely to be used later). 


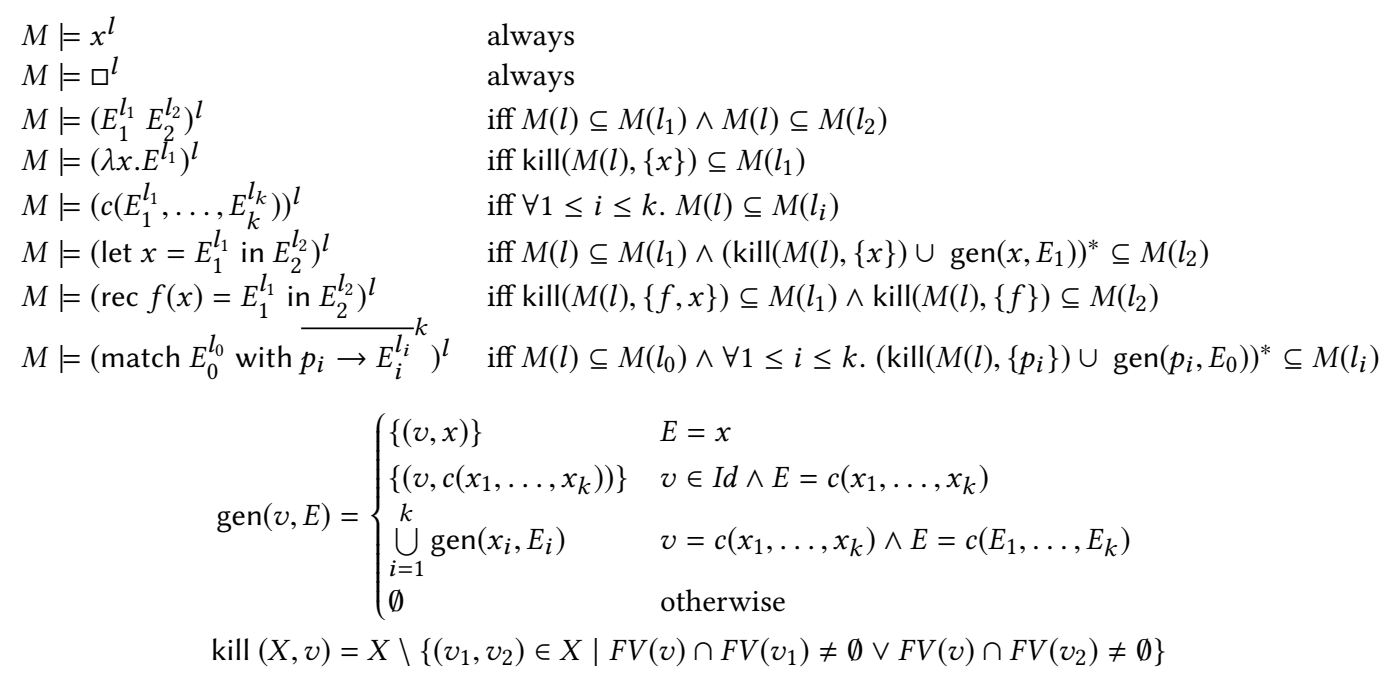

Fig. 7. Data-flow analysis for components reduction

Reducing Syntax Components. In addition to reducing variable components, we reduce syntax components as well. Note that our full language in Appendix B is too large to perform exhaustive search. For instance, the language has 35 different syntactic cases for expressions. To reduce the search space more effectively, we used a simple heuristic that only searches through the syntax components that appear in the correct implementation provided by an instructor. Given the correct implementation $E_{c}$, we extract its components using $\mathrm{E}: \operatorname{Exp} \rightarrow \wp(\operatorname{Exp})$ as follows:

$$
\begin{gathered}
\mathrm{E}(x)=\emptyset \quad \mathrm{E}(\lambda x . E)=\{\lambda x . \square\} \cup \mathrm{E}(E) \quad \mathrm{E}\left(E_{1} E_{2}\right)=\{\square \square\} \cup \mathrm{E}\left(E_{1}\right) \cup \mathrm{E}\left(E_{2}\right) \\
\mathrm{E}\left(\text { let } x=E_{1} \text { in } E_{2}\right)=\{\text { let } x=\square \text { in } \square\} \cup \mathrm{E}\left(E_{1}\right) \cup \mathrm{E}\left(E_{2}\right) \quad \mathrm{E}\left(c\left(E_{1}, \ldots, E_{k}\right)\right)=\cup_{i=1}^{k} \mathrm{E}\left(E_{i}\right) \\
\mathrm{E}\left(\text { letrec } f(x)=E_{1} \text { in } E_{2}\right)=\{\text { letrec } f(x)=\square \text { in } \square\} \cup \mathrm{E}\left(E_{1}\right) \cup \mathrm{E}\left(E_{2}\right) \\
\mathrm{E}\left(\text { match } E \text { with } p_{1} \rightarrow E_{1}|\cdots| p_{k} \rightarrow E_{k}\right)=\left\{\text { match } \square \text { with } p_{1} \rightarrow \square|\cdots| p_{k} \rightarrow \square\right\} \cup \mathrm{E}(E) \cup \cup_{i=1}^{k} \mathrm{E}\left(E_{i}\right)
\end{gathered}
$$

Our algorithm uses the function SyntaxComponents $\left(E_{c}\right)=\mathrm{E}\left(E_{c}\right)$ at line 2 of Algorithm 1 .

\subsection{Pruning by Symbolic Execution}

The final component of our algorithm is pruning based on symbolic execution.

Motivation. Consider the following partial program:

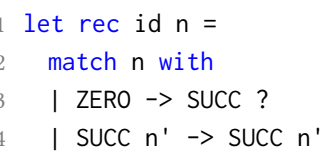

Suppose that we have an input-output testcase $\{$ ZERO $\mapsto$ ZERO , which means that the function id on ZERO must evaluate to ZERO. Note that although the partial program has no type error, it will never match the given input-output behavior because SUCC e cannot be equal to ZERO regardless of the expression e. We detect such semantic discrepancies by running a symbolic executor on the partial programs encountered during the search algorithm.

Symbolic Execution Rules. We designed a symbolic executor that efficiently detects partial programs that are inconsistent with respect to the given testcases. The semantics for symbolic 


$$
\begin{aligned}
& \overline{\widehat{\rho} \vdash x \Rightarrow \widehat{\rho}(x)} \quad \overline{\widehat{\rho} \vdash \square \Rightarrow \beta} \text { new } \beta \quad \frac{\hat{\rho} \vdash \lambda x \cdot E \Rightarrow(x, E, \widehat{\rho})}{\widehat{\hat{\rho}} \vdash c\left(E_{1}, \ldots, E_{k}\right) \Rightarrow c\left(\widehat{v_{1}}, \ldots, \widehat{v_{k}}\right)} \\
& \frac{\widehat{\rho} \vdash E_{1} \Rightarrow \beta}{\widehat{\rho} \vdash E_{1} E_{2} \Rightarrow \beta^{\prime}} \text { new } \beta^{\prime} \quad \frac{\hat{\rho} \vdash E_{1} \Rightarrow\left(x, E^{\prime}, \widehat{\rho}^{\prime}\right) \quad \hat{\rho} \vdash E_{2} \Rightarrow \widehat{v} \quad \widehat{\rho}^{\prime}[x \mapsto \widehat{v}] \vdash E^{\prime} \Rightarrow \widehat{v^{\prime}}}{\hat{\rho} \vdash E_{1} E_{2} \Rightarrow \widehat{v^{\prime}}} \\
& \frac{\widehat{\rho} \vdash E_{1} \Rightarrow\left(f, x, E^{\prime}, \widehat{\rho}^{\prime}\right) \quad \widehat{\rho} \vdash E_{2} \Rightarrow \widehat{v} \quad \hat{\rho}^{\prime}\left[x \mapsto \widehat{v}, f \mapsto\left(f, x, E^{\prime}, \widehat{\rho}^{\prime}\right)\right] \vdash e^{\prime} \Rightarrow \widehat{v^{\prime}}}{\widehat{\rho} \vdash E_{1} E_{2} \Rightarrow \widehat{v^{\prime}}} \\
& \frac{\widehat{\rho}\left[f \mapsto\left(f, x, E_{1}, \widehat{\rho}\right)\right] \vdash E_{2} \Rightarrow \widehat{v}}{\widehat{\rho} \text { ค letrec } f(x)=E_{1} \text { in } E_{2} \Rightarrow \widehat{v}} \quad \frac{\widehat{\rho} \vdash E \Rightarrow \widehat{v} \quad \widehat{v} \text { includes symbols }}{\widehat{\rho} \vdash \text { match } E \text { with } p_{1} \rightarrow E_{1}|\cdots| p_{k} \rightarrow E_{k} \Rightarrow \beta} \text { new } \beta \\
& \frac{\widehat{\rho} \vdash E \Rightarrow \widehat{v} \quad \widehat{\operatorname{match}}\left(\widehat{v}, p_{i}\right) \quad \forall j<i . \neg \widehat{\operatorname{match}}\left(\widehat{v}, p_{j}\right) \quad \widehat{\operatorname{bind}}\left(\widehat{\rho}, p_{i}, \widehat{v}\right) \vdash E_{i} \Rightarrow \widehat{v}_{i}}{\widehat{\rho} \vdash \operatorname{match} E \text { with } p_{1} \rightarrow E_{1}|\cdots| p_{k} \rightarrow E_{k} \Rightarrow \widehat{v}_{i}}
\end{aligned}
$$

Fig. 8. Symbolic execution rules for pruning search space. $\widehat{\operatorname{match}}(v, p)=\left(p=c\left(\widehat{v}_{1}, \ldots, \widehat{v}_{k}\right)\right) \wedge(p=$ $\left.\left.c\left(x_{1}, \ldots, x_{k}\right)\right) . \widehat{\operatorname{bind}}\left(\rho, c\left(x_{1}, \ldots, x_{k}\right), c\left(\widehat{v}_{1}, \ldots, \widehat{v}_{k}\right]\right)\right)=\left[x_{i} \mapsto \widehat{v}_{i}\right]_{i=1}^{k}$.

execution is an extension of the concrete semantics. We first extend the value domain ( $\mathrm{Val})$ to include symbolic values $($ Symbol $): \widehat{\text { Val }}=$ Symbol $+\widehat{\text { Cnstr }}+\widehat{\text { Closure }}+\widehat{\text { RecClosure }}$, where $\widehat{\text { Cnstr }}=I d \times \widehat{\text { Val }}$, $\widehat{\text { Closure }}=I d \times E x p \times \widehat{E n v}$, and RecClosure $=I d \times I d \times E x p \times \widehat{E n v}$. A symbolic store $\widehat{\rho} \in \widehat{E n v}=I d \rightarrow \widehat{\text { Val }}$ maps variables to their symbolic values. Let $\mathcal{S}(E): \widehat{E n v} \rightarrow \widehat{V a l}$ be the symbolic executor based on the rules defined in Fig. 8. Whenever the executor encounters a hole $\square$, it assigns a fresh symbol $\beta$. In the application case $\left(E_{1} E_{2}\right)$ where the function expression $E_{1}$ is not yet a complete expression (i.e. $\hat{\rho} \vdash E_{1} \Rightarrow \beta$ ), the executor assigns a new symbol. In the pattern matching case, when symbols are involved in the expression $E$, the executor avoids complex reasoning and aggressively abstracts the final result to a fresh symbol. The remaining rules are analogous to the standard concrete semantics, except that the executor manipulates symbols.

Pruning using Symbolic Execution. Now we explain how we perform pruning with the symbolic executor. Given a partial program $E$ (with an input variable $x$ ) and a set $\mathcal{T}$ of testcases, we first generate the following formula $\phi_{E, \mathcal{T}}$ by running the symbolic executor on $E$ for each testcase $\left(v_{i}, v_{o}\right) \in \mathcal{T}: \phi_{E, \mathcal{T}}=\bigwedge_{\left(v_{i}, v_{o}\right) \in \mathcal{T}}$ equal $\left(\mathcal{S}(E)\left[x \mapsto v_{i}\right], v_{o}\right)$, where the function equal relates the input and output values and produces a formula as follows:

$$
\begin{aligned}
\text { equal }\left(\beta, c\left(\widehat{v}_{1}, \ldots, \widehat{v}_{k}\right)\right) & =\left(\beta=c\left(\widehat{v}_{1}, \ldots, \widehat{v}_{k}\right)\right)(\beta \in \mathbb{S}) \\
\text { equal }\left(c\left(\widehat{v}_{1}, \ldots, \widehat{v}_{k}\right), c^{\prime}\left(\widehat{v}_{1}^{\prime}, \ldots, \widehat{v}_{k}^{\prime}\right)\right) & =\left(c=c^{\prime}\right) \wedge \bigwedge_{1 \leq i \leq k} \text { equal }\left(\widehat{v}_{i}, \widehat{v}_{i}^{\prime}\right) \\
\text { equal }\left({ }_{-},-\right) & =\text {false }
\end{aligned}
$$

Then, we prune out the program $E$ if and only if $\phi_{E, \mathcal{T}}$ is unsatisfiable:

$$
\text { Inconsistent }(E, \mathcal{T}) \Longleftrightarrow \phi_{E, \mathcal{T}} \text { is unsatisfiable }
$$

which can be checked using an off-the-shelf SMT solver.

\section{EVALUATION}

In this section, we evaluate FIxML aiming at answering the following research questions:

- Effectiveness: How effectively can FixML correct real student submissions? Is it applicable to a wide range of functional programming assignments? Is it efficient enough to provide quick feedback to students? 
Table 1. Performance of FIXML on 497 student submissions. \#P reports the number of target programs for each problem. \#T reports the number of testcases used for each problem. LOC reports the number of lines in submissions averaged over all submissions as well as the LOCs of the smallest and largest submissions. Time reports average time took for localizing and fixing errors in seconds. Fix Rate reports the ratio of successfully fixed programs by FIxML.

\begin{tabular}{|c|c|c|c|c|c|c|}
\hline No & Problem Description & $\# \mathrm{P}$ & $\# \mathrm{~T}$ & $\begin{array}{c}\text { LOC } \\
(\min -\max )\end{array}$ & Time & $\begin{array}{c}\text { Fix Rate } \\
\text { (\#Fix) }\end{array}$ \\
\hline 1 & Filtering elements satisfying a predicate in a list & 3 & 10 & $6 \quad(6-7)$ & 13.0 & $100 \% \quad(3)$ \\
\hline 2 & Finding a maximum element in a list & 32 & 10 & $8(4-14)$ & 0.2 & $100 \%(32)$ \\
\hline 3 & Mirroring a binary tree & 9 & 10 & $10(9-14)$ & 0.1 & $89 \% \quad(8)$ \\
\hline 4 & Checking membership in a binary tree & 15 & 17 & $11(9-18)$ & 5.2 & $80 \%(12)$ \\
\hline 5 & Computing $\sum_{i=j}^{k} f(i)$ for $j, k$, and $f$ & 23 & 11 & $5 \quad(2-9)$ & 4.2 & $78 \%(18)$ \\
\hline 6 & Adding and multiplying user-defined natural numbers & 34 & 10 & $20(10-50)$ & 20.6 & $59 \%(20)$ \\
\hline 7 & Finding the number of ways of coin-changes & 9 & 10 & $21(6-35)$ & 2.6 & $44 \% \quad(4)$ \\
\hline 8 & Composing functions & 28 & 12 & $7(1-19)$ & 5.5 & $43 \%(12)$ \\
\hline 9 & Implementing a leftist heap using a priority queue & 20 & 13 & $43(33-72)$ & 2.6 & $40 \% \quad(8)$ \\
\hline 10 & Evaluating expressions and propositional formulas & 101 & 17 & $32(17-90)$ & 1.2 & $39 \%(39)$ \\
\hline 11 & Adding numbers in user-defined number system & 14 & 10 & $52(19-138)$ & 7.0 & $36 \%(5)$ \\
\hline 12 & Deciding lambda terms are well-formed or not & 86 & 11 & $28(13-79)$ & 1.3 & $26 \%(22)$ \\
\hline \multirow[t]{2}{*}{13} & Differentiating algebraic expressions & 123 & 17 & $36(14-154)$ & 11.4 & $25 \%(31)$ \\
\hline & Total / Average & 497 & 158 & $27(2-154)$ & 5.4 & $43 \%(214)$ \\
\hline
\end{tabular}

- Helpfulness: How helpful is FixML for students? How often do students produce logical errors in functional programming problems? How difficult is it to correct those errors by themselves? Does FIxML help students to better understand the errors?

- Utility of Techniques: How useful is the combination of various techniques in Section 4? Does it significantly outperform simpler approaches?

FIXML is written with $\sim 6400$ lines of OCaml code. We used the Alt-Ergo Zero SMT solver ${ }^{3}$ to check the satisfiability of constraints generated by symbolic execution (Section 4.4). All experiments were done on an iMac with Intel i5 CPU and 16GB memory.

\subsection{Effectiveness}

Benchmarks. We have evaluated the effectiveness of FIxML with 497 programs with logical errors collected from 13 functional programming assignments used in a Programming Languages course over the last few years. Those programs have logical errors only, not syntax or type errors

The problem descriptions are presented in Table 1. We classify the benchmark problems into introductory (\#1-\#5), intermediate (\#6-\#9), and advanced (\#10-\#13) problems. The problems at introductory-level let students to experience basic functional programming concepts such as recursion, inductive datatypes, and high-order functions. Solving intermediate problems needs relatively more challenging programming skills. The advanced problems require familiarity in programming language concepts or algorithmic skills. For example, problem \#10 asks to write a simple interpreter for a language with arithmetic expressions and propositional formulas and problem \#12 requires to understand the concept of lambda calculus.

Note that our benchmark problems are comparatively more challenging than those typically used in prior work. Previous feedback generation systems mostly focus on problems at introductory-level

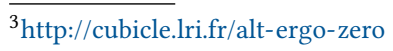


onl, such as programming tasks that manipulate integers or arrays (e.g. reversing numbers, finding the $k$ th largest element, etc) [Gulwani et al. 2018; Singh et al. 2013; Wang et al. 2018]. In this paper, we aim for more sizable programs up to 100 lines of code.

In Table 1, we report the number of incorrect submissions we could collect for each problem $(\# \mathrm{P})$, the number of testcases (\#T), (average, smallest, largest) LOCs of the submissions (LOC), the average time took to generate corrections (including localization), and the ratio of successfully fixed submissions for each problem with respect to the entire programs (Fix Rate).

Results. The results in Table 1 indicate that FixML is powerful and capable of fixing logical errors in real student submissions. In summary, FIxML successfully fixed 214 out of 497 submissions in 5.4 seconds on average. ${ }^{4}$ For introductory-level problems (\#1-\#5), FixML fixed most (89\%, 73/82) of the submissions in 2.5 seconds on average. For intermediate-level problems (\#6-\#9), the fix rate was $48 \%$ (44/91) and the average time for fixing was 11.6 seconds. For problems at advanced level (\#10-\#13), FixML was able to correct 30\% (97/324) in 4.8 seconds on average. Although the fix rates decrease in problems at advanced-level, the results are still impressive, considering the size and complexity of the benchmark programs. For example, FIxML managed to accurately localize and repair an error in the largest submission (154 lines) presented in Appendix C.

In Table 1, all the generated patches are correct; the patched programs are semantically equivalent to the reference programs. In test-based repair, generated patches are likely to overfit the given testcases [Smith et al. 2015]. In this work, we avoided this issue by manually refining the testcases until the repaired programs become semantically equivalent to the reference implementation. We started with 130 testcases (10 testcases for each problem), which resulted in 58 overfitted patches (27\%), and increased the set to 158 testcases through the refinement process. The more detailed discussion is included in Section 5.4.

Moreover, we have manually checked the quality of the patches. Even though the repaired programs are semantically correct, they might not be ideal for various reasons (e.g., readability, optimality, etc.). We found that 25 out of 214 are not ideal. However, 23 of them could be easily transformed into ideal ones via simple postprocessing. For example, consider the function mem (Problem 4 in Table 1) written by a student, which is supposed to check whether $n$ is stored in the given binary tree (tree):

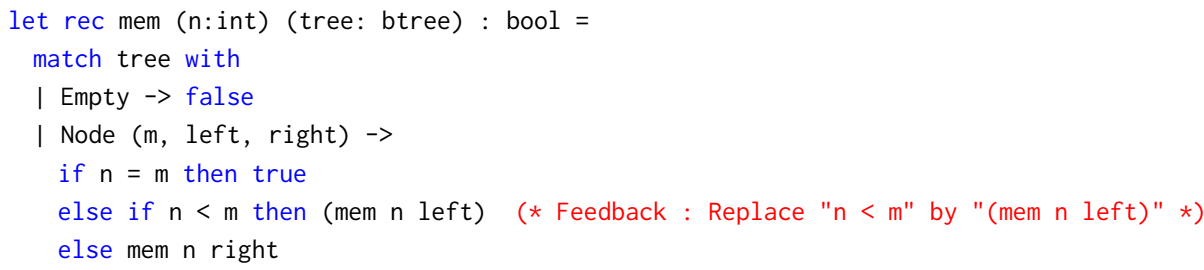

The program has an error at line 6, where the student misunderstood that a binary search tree, not a plain binary tree, was given. FixML corrected the conditional expression $(n<m)$ by (mem $n$ left), which checks the membership in both left and right subtrees. The patch, however, is undesirable since the (mem $n$ left) is redundant. In this case, the fix can be modified through post-processing, which replaces "if (mem $n$ left) then mem $n$ left else mem $n$ right" by "(mem $n$ left) II (mem n right)" based on the fact that the conditional expression is syntactically equivalent to the true branch. The remaining two cases were not ideal because of the imprecision of error localization. We describe this case in the first paragraph ("Accurate Localization") of Section 5.4.

\footnotetext{
${ }^{4}$ We manually checked the correctness of the 214 fixes.
} 


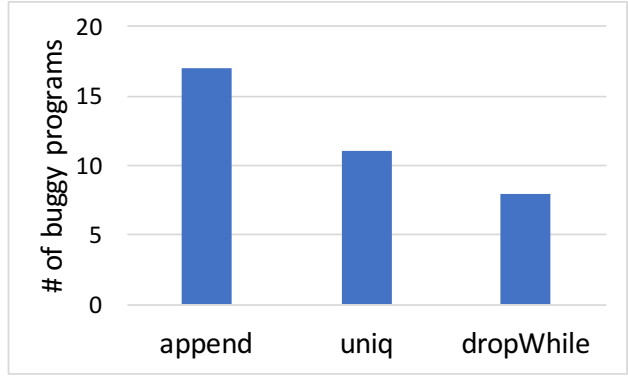

(a) The number of the buggy submissions

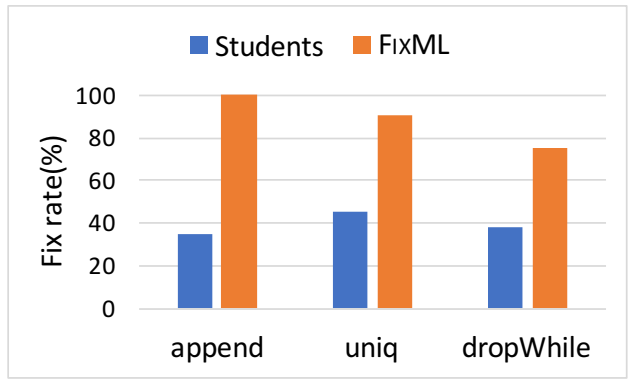

(b) Fix rates by students and FIXML

Fig. 9. User study results

\subsection{Helpfulness}

To assess how helpful FIxML is for students, we have conducted user study with 18 undergraduate students who took the Programming Language course taught by the authors.

Scenario. The user study proceeded for one hour and consisted of three parts. First, we asked the students to solve the three programming problems not included in the problem set of the course:

(1) Problem 1 (append): Write a function, which takes two lists and appends the first list to the second one while removing duplicating elements (Example 3 in Section 2.1).

(2) Problem 2 (unique): Write a function, which removes duplicated elements from a given list so that the list contains unique elements. For instance, given input $[5 ; 6 ; 5 ; 4]$ the expected output is $[5 ; 6 ; 4]$.

(3) Problem 3 (dropWhile): Write a function, which takes a predicate and a list, and removes the elements of the list until an element on which the predicate is false is found. For example, dropWhile (fun $x \rightarrow x \bmod 2=0$ ) $[2 ; 4 ; 7 ; 6 ; 9]$ outputs $[7 ; 6 ; 9]$.

Because solving all of the problems from scratch with the limited time is a bit challenging, we provided templates for each problem. The templates include helper functions and students are allowed to use them to complete the programs. For example, we used the following template for Problem 2:

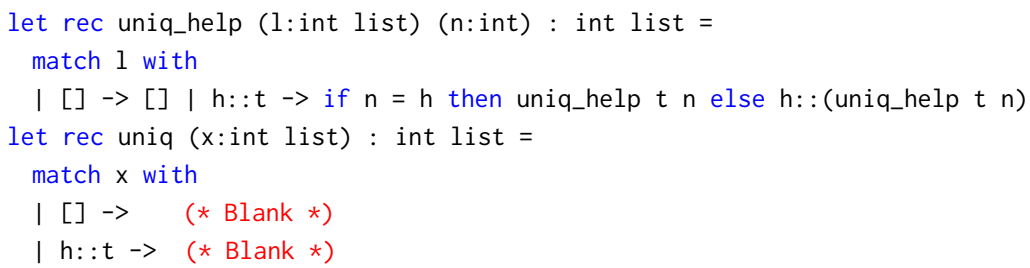

where the job of the students is to replace the two blanks by appropriate expressions. We collected the answers by students and counted the number of incorrect submissions for each problem.

In the second part of the user study, students were asked to fix their errors by themselves using counterexamples provided by the instructor. The last task is for students to use FIxML and evaluate it based on how useful FIxML is to better understand their mistakes. The survey questions used for tool evaluation are in Table 2.

Results. Fig. 9 summarizes the results from the first and second parts of user study. Fig. 9a shows that most of the students felt difficulty in solving the problems, even though templates 
Table 2. Questions used in the survey and corresponding responses from 18 undergraduates. Q1 targeted 12 students who had succeeded in correcting their errors by themselves for at least one problem. Q2 targeted 12 students who failed to fix their errors by themselves for at least one problem. Q3 targeted all 18 students. ( $n$ ) shows the number of actual responses.

\begin{tabular}{|l|c|c|c|}
\hline Question & Yes & Neutral & No \\
\hline $\begin{array}{c}\text { Q1. Compared to your own corrections, } \\
\text { does FIxML generate better corrections? }\end{array}$ & $67 \%(8)$ & $33 \%(4)$ & $0 \%(0)$ \\
\hline $\begin{array}{c}\text { Q2. Does FixML help to understand your mistakes } \\
\text { when you cannot resolve them by yourself? }\end{array}$ & $50 \%(6)$ & $42 \%(5)$ & $8 \%(1)$ \\
\hline $\begin{array}{c}\text { Q3. Is FixML overall useful in learning } \\
\text { functional programming? }\end{array}$ & $72 \%(13)$ & $28 \%(5)$ & $0 \%(0)$ \\
\hline
\end{tabular}

were given. In Problem 1 (append), 94\% (17/18) of the students failed to correctly implement the desired functionality. Even in the best case (dropWhile), 44\% (8/18) of the students submitted buggy programs. All submitted programs are free of syntax and type errors and only contain logical errors.

Fig. 9b shows that providing error-triggering testcases only does not much help students to figure out their mistakes. Most students failed to fix their errors by themselves using the provided testcases, although we allowed them to use additional time as long as they requested. At best, $45 \%$ of the students were able to correct their errors (Problem 2 (uniq), 5/11). On the other hand, FIxML was able to correct most of the errors introduced by the students. Remarkably, FIxML successfully corrected all of the errors for Problem 1 (append).

The results in Table 2 shows that FixML can be overall useful for students. We let students to experience FIxML and evaluate how helpful it is in assisting diagnosing and understanding their mistakes. We asked three questions that aim to assess the following aspects: (Q1) qualities of the feedback by FixML; (Q2) helpfulness in understanding their mistakes; and (Q3) overall usefulness in learning functional programming. For Q3, we asked students to additionally justify their responses.

For Q1, we targeted 12 students who succeeded in correcting their errors by themselves for at least one problem. All of them recognized that FixML provides corrections of better or similar qualities compared to their own ones. Indeed, from further inspections of the students' corrections, we found that the students' corrections are often verbose (e.g. defining multiple redundant functions that have the same functionality) and introduced new errors as they are likely to produce corrections overfit to the testcases given by the instructor. On the other hand, the FixML-generated corrections were minimal and easy-to-understand.

For Q2, we targeted 12 students who failed to fix their errors by themselves for at least one problem. Among 12 students, only one responded that FIxML did not help to understand her mistakes, while the rest 11 answered that they were able to understand the reasons for their mistakes based on FIxML's corrections.

For Q3, we targeted all 18 students. 72\% of them agreed that FIXML is definitely useful for learning functional programming with the following comments: "FIXML can play a role as an automated teacher by pinpointing failure points and providing corresponding corrections", "Students can immediately resolve their troubles thanks to the realtime-feedback of FIxML", "FIxML's corrections are concise and valuable for enhancing programming skills", etc. The $28 \%$ of students (5 students) in Q3 overall acknowledged the usefulness of FIxML, but responded 'Neutral' due to the following reasons. Two of them worried that students are likely to become dependent on FixML without trying to solve the problems on their own. Other two students replied that they may be able to fix 


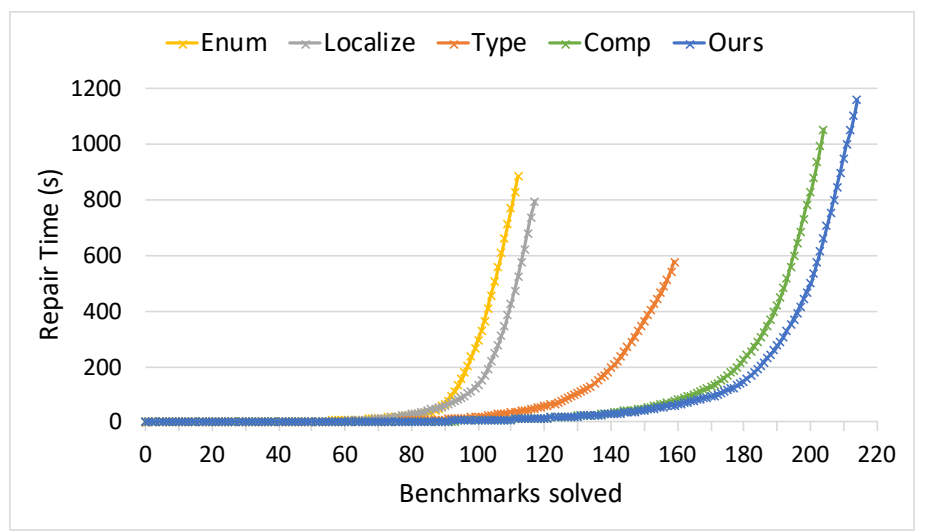

Fig. 10. Utility of techniques

errors by themselves in most cases without FIxML. The last one responded that FIxML may not be able to help students who have difficulties in designing algorithms at early stages.

\subsection{Utility of Techniques}

FixML could not be succesful without the combination of the techniques presented in Section 4. In this section, we justify this claim by evaluating the impact of major techniques used in FIxML.

In Fig. 10, the line labelled 'Ours' depicts the cumulative repair time of FixML when we applied all techniques (statistical error localization, type-directed search, component reduction, and pruning by symbolic execution). 'Comp' represents the performance of FixML except the symbolic execution (statistical error localization, component reduction, type-directed search). 'Type' shows the result for FIXML with statistical error localization and type-directed search, which may provide a glimpse of the current state-of-the-art functional program synthesizers [Feser et al. 2015; Frankle et al. 2016; Osera and Zdancewic 2015; Polikarpova et al. 2016] whose key technology is type-based pruning. 'Localize' shows the performance of the algorithm that uses statistical error localization and plain enumerative search without type-based pruning. 'Enum' represents the performance of FIXML when it performs plain enumerative search but does not perform statistical reasoning in localizing errors (i.e., the suspicious score in Section 4.1 is computed as $\operatorname{score}(e)=\operatorname{size}(e))$ ). For each submission, time limit for repair was one-minute.

The results show that the combination of all techniques ('Ours') remarkably improves the performance of the type-directed search ('Type') on both effectiveness (i.e. the number of successful fixes) and efficiency (i.e. the repair time when compared for the same number of benchmarks). Specifically, while 'Type' corrected only 160 submissions, 'Ours' corrected 214 submissions. In terms of efficiency, while 'Type' spent 579 seconds for fixing the first 160 submissions, 'Ours' do so for the same set of submissions in 65 seconds.

The competitiveness of 'Ours' over 'Type' is noteworthy, because the type-directed search itself is already well-tuned compared to 'Localize': 'Localize' was able to repair only 118 submissions and it took 796 seconds to do so, whereas 'Type' only required 50 seconds to fix the same 118 submissions.

Additionally, the result shows that both component reduction and symbolic execution have significant impacts on the performance. By comparing 'Comp' to 'Type' in Fig. 10, component reduction made it possible to fix 45 more submissions and only spent 78 seconds to fix 160 patches, whereas 'Type' spent 579 seconds to fix the same number of programs. Even though the component 
reduction remarkably speed up the tool, symbolic execution can still enhance FixML more. 'Ours' repaired 214 submissions while 'Comp' corrected 205 submissions. Furthermore, 'Ours' took 706 seconds to fix 205 programs in contrast to 'Comp' which required 1052 seconds.

Also, the difference between 'Localize' and 'Enum' tells that applying statistical error localization is useful for accelerating the repair time. 'Enum' repaired 113 programs, while 'Localize' repaired 118 programs. Furthermore, while 'Enum' spent 884 seconds for repairing 113 programs, 'Localize' only spent 524 seconds for repairing the same set of the programs. Note that, since our localization procedure is currently based on simple statistical reasoning, there still exists a lot of room for improvements. We will discuss more details in Section 5.4

\subsection{Discussion 1: Limitations and Future Work}

In experiments, a number limitations of FIxML has been identified. Accordingly, we plan to improve FIxML in the following directions.

Accurate Localization. More accurate error-localization is needed to produce better corrections. For example, consider the filter function (Problem 1 in Table 1) written by a student:

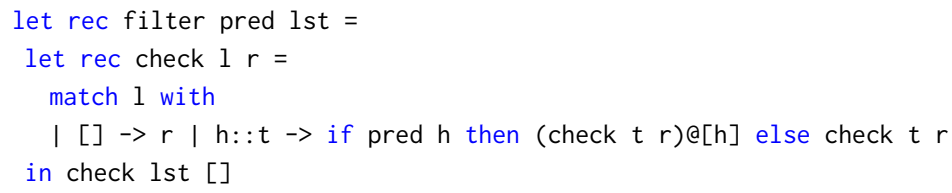

The student implemented filter by defining a helper function check and invoking it. Function check has a bug at line 4 , where it appends the head (h) at the end of the list. As a result, for example, filter (fun $x \rightarrow x$ mod $2=0$ ) $[1 ; 2 ; 3 ; 4]$ produces $[4 ; 2]$, not $[2 ; 4]$. To fix the bug, the expression (check $t r$ ) $@[h]$ should be replaced by $h:$ : (check $t r$ ). However, FixML pointed the call expression (check lst []) at line 5 as the error location and fixed the error by replacing the expression by (check (check lst []) []). Although this is a semantically correct fix, it might not be ideal feedback for students.

Fixing Multiple Errors. Currently, FixML focuses on fixing programs with a single error location. Thus, it fails to produce corrections for the following submission (Problem 11 in Table 1):

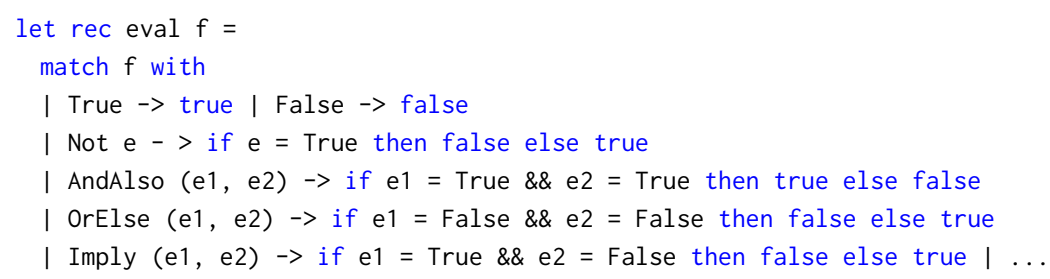

where eval takes a propositional formula $f$ and intends to evaluate its truth value using recursion. However, the implementation is buggy as it consistently missing recursive calls in all necessary places; the expressions True, False, e, e1, and e2 at lines 4-7 must be replaced by true, false, eval e, eval e1, and eval e2, respectively. We plan to enable FixML to iteratively fix similar bugs in multiple locations.

Automatic Testcase Generation. To improve usefulness, we realized that FixML should be combined with automatic testcase generation, as its effectiveness depends on the quality of given testcases. For example, consider the function sigma (Problem 5 in Table 1) written by a student:

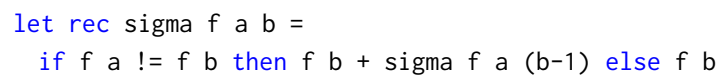




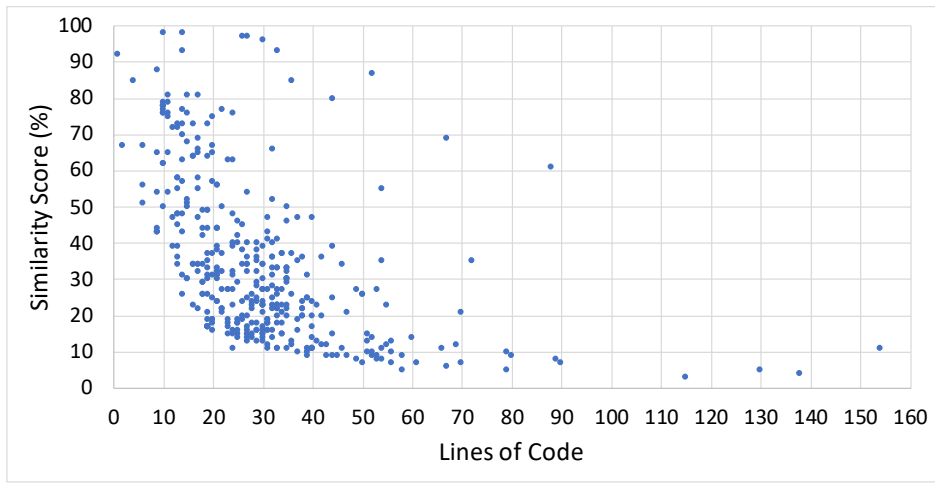

Fig. 11. Similarity between correct and incorrect submissions

Given $f$, $a$, and b, sigma $f a b$ should compute $\sum_{i=a}^{b} f(i)$. The implementation works correctly as long as $f$ is injective (e.g. $f=$ fun $x \rightarrow x * x)$. However, it becomes incorrect when, for example, $f$ is (fun $x \rightarrow x$ mod 3 ). The bug is at line 2, where the condition $f a !=f b$ must be replaced by a $!=b$. At an early stage of this work, FIxML was not able to find this error, as such a counterexample was missing in our testcases. We plan to automatically generate high-quality testcases, which then enables FixML not to miss tricky bugs.

Scalability. Although we have improved scalability significantly, FIxML still needs more advanced program synthesis techniques to generate complex repairs. For instance, FixML could not fix the student's submission below (Problem 13 in Table 1):

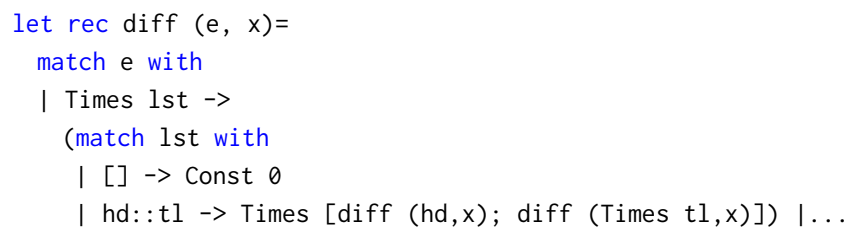

where a desirable fix is to replace Times [diff (hd, $x$ ); diff (Times $t l, x)$ ] (i.e., $(f g)^{\prime}=f^{\prime} g^{\prime}$ ) at line 6 with Sum [Times ( $(\operatorname{diff}(h d, x)):: t l)$; Times [hd; diff (Times tl,x)]] (i.e., $\left.(f g)^{\prime}=f^{\prime} g+f g^{\prime}\right)$. However, FixML failed to produce the correction even after 15 minutes. We plan to develop more powerful synthesis techniques to generate sizable repairs.

\subsection{Discussion 2: Similarity between Correct and Incorrect Submissions}

A recent trend in automatic feedback generation systems is data-driven approach [Gulwani et al. 2018; Pu et al. 2016; Wang et al. 2018], where a number of correct implementations that are similar to a given buggy program are exploited to find appropriate candidates for corrections. Readers might wonder FIxML can benefit from this promising and complementary approach. To answer this question, we analyzed similarities between correct and incorrect programs submitted for our 13 benchmark problems in Table 1.

The analysis result in Fig. 11 implies that although the data-driven approach can be effective for introductory-level problems, it would be unsuitable for more complex programs. In this study, we checked whether we could find any similar programs for each incorrect submission using Moss [Schleimer et al. 2003], a system for quantifying similarities between programs. ${ }^{5}$ For each

\footnotetext{
${ }^{5}$ For OCaml, we used the implementation available at https://github.com/Chris00/ocaml-moss
} 
erroneous submission, we provided Moss with the buggy program and a set of correct submissions submitted for the same problem. Then, Moss ranks the correct submissions based on the similarity score with respect to the buggy program. We repeated this process for each incorrect submission $P$, and stored $(P, s)$, where $s$ is the score of the correct program with the highest similarity. In total, we used 385 incorrect programs and 4350 correct programs. ${ }^{6}$ Fig. 11 depicts the data obtained from this study, where for each $(P, s)$, the $\mathrm{x}$-axis shows the size of $P$ and y-axis reports $s$. The result indicates that we can hardly find similar correct submissions as the program size increases. Specifically, for the programs larger than 20 lines (266 among 385), which include most of our intermediate and advanced benchmarks, $85 \%$ (226/266) of them had highest similarity scores less than 40 . According to our experience, the similarity score 40 means that the two programs are substantially different from each other. That is, we were frequently unable to find useful correct programs similar to a given incorrect program.

\section{RELATED WORK}

In this section, we survey recent researches that are closely related to our work.

Debugging functional programs. For functional languages, prior works have focused on localizing and fixing type errors [Chen and Erwig 2014; Lerner et al. 2007; Pavlinovic et al. 2014, 2015; Seidel et al. 2017; Wu et al. 2017; Wu and Chen 2017; Zhang et al. 2017]. For example, Seidel et al. [2017] recently proposed a data-driven approach to localize type errors in OCaml programs. Wu et al. [2017]; Wu and Chen [2017] studied how type errors are fixed and presented LEARNSKELL for producing user-friendly feedback on type errors. Pavlinovic et al. [2014, 2015] presented SMT-based techniques for localizing type errors. Chen and Erwig [2014] presented a method for fixing type errors by generating possible changes and checking the them using type checker. Zhang et al. [2017] present a technique for localizing errors detected by static analyses such as type system. Unlike these works, our goal is to localize and fix logical errors in functional programs. The technique by Kneuss et al. [2015] is able to repair logical error of functional programs but it requires user-provided specifications.

Automated feedback generation. Recently, a large amount of work has been devoted to providing feedback on logical errors [D'Antoni et al. 2016; Gulwani et al. 2018; Kim et al. 2016; Pu et al. 2016; Singh et al. 2013; Wang et al. 2018], type errors [Seidel et al. 2017; Wu et al. 2017; Wu and Chen 2017], syntax errors [Bhatia et al. 2018; Gupta et al. 2017], and performance problems [Gulwani et al 2014] in students' programs. AutoGrader [Singh et al. 2013], which repairs students' programs using predetermined correction rules provided by instructors, has inspired recent advances in automatic feedback generation. QLose [D'Antoni et al. 2016] aims to generate repairs that are optimal in that the resulting repaired program is most similar to the original buggy program. APEX [Kim et al. 2016] provides explanations for root causes of logical errors in students' programs, but does not attempt to repair the errors. Recent data-driven approach, which exploits a number of correct programs to find appropriate candidates for corrections, has shown to be effective to introductory-level programming problems [Gulwani et al. 2018; Pu et al. 2016; Wang et al. 2018]. Our work lies in this line of research but our goal and techniques are different from those in existing works: 1) FIxML is the first system aiming for fixing logical errors in functional programs while most feedback generation systems are for imperative languages, and 2) we present an error-correction algorithm based on statistical localization and enhanced type-directed synthesis, which is able to accurately produce useful feedback beyond introductory-level problems.

${ }^{6}$ Among the 497 incorrect submissions in Table 1, we failed to run Moss on 112 programs. 
Program synthesis. Program synthesis has been used in many application domains such as string-processing [Gulwani 2011; Kini and Gulwani 2015], data processing [Feng et al. 2017a], complex APIs [Feng et al. 2017b], database [Yaghmazadeh et al. 2017], and many others. Our work uses program synthesis for generating feedback on functional programs.

In particular, our work leverages recent advances in functional program synthesis, where type system has played a key role for accelerating functional program synthesizers by pruning illtyped programs from the search space [Feser et al. 2015; Frankle et al. 2016; Osera and Zdancewic 2015; Polikarpova et al. 2016]. However, in our experience, the type-based pruning alone was not enough to be scalable, hence we applied two additional techniques: reduction of semantically redundant components and pruning search space using symbolic execution. There are also some other functional program synthesizers. Escher [Albarghouthi et al. 2013] performs heuristic goaldirected search, where a final solution is constructed by combining subsolutions with if-then-else statement. The proposed approach is hard to be generalized into a significant subset of OCaml that we target. Leon [Kneuss et al. 2013] aims to synthesize recursive functional programs verified with proofs, where specifications should be given as predicates in first-order formula unlike ours.

Techniques used by So and Oh [2017] and Balog et al. [2017] would help to improve performance of our system. So and Oh [2017] use abstract interpretation for pruning search space in the context of imperative program synthesis. Balog et al. [2017] use deep learning to optimize cost models used in search-based synthesis. We believe that using abstract interpretation and machine learning can significantly improve our results, which we leave as future work.

Error localization. The researchers in [Jose and Majumdar 2011] proposed a localization method based on MAX-SAT problem solving. However, the proposed method may not be directly applicable to our setting, because it is hard to generate constraints on high-order functions precisely and statically. Instead, we adapt a well-known statistical fault localization method [Ball et al. 2003; Griesmayer et al. 2007; Groce et al. 2006; Jones et al. 2002; Renieris and Reiss 2003] for functional programs.

Automatic program repair. Aside from the researches for repairing students' programs, there also have been many recent researches for fixing bugs in large software, e.g., [Forrest et al. 2009; Kim et al. 2013; Kneuss et al. 2015; Könighofer and Bloem 2011; Le Goues et al. 2012; Long and Rinard 2016; Nguyen et al. 2013; Weimer et al. 2009]. The techniques for repairing students' programs and developers' software are complementary; while the former can generate relatively complicated patches for small programs, the latter usually produces small patches for large programs. For example, SEMFIX [Nguyen et al. 2013] focuses on fixing righthand-side of assignments or branch conditions for scalability.

\section{CONCLUSION}

In this paper, we presented FIxML, the first system for automatically diagnosing and correcting logical errors in functional programming assignments. We presented the new error-correction algorithm that combines statistical error-localization, type-directed enumeration, automatic component reductions, and pruning via symbolic execution. We have demonstrated the effectiveness of our approach with 497 students' submissions and the usefulness with 18 undergraduate students.

\section{ACKNOWLEDGMENTS}

We thank Jaehyun Lim for his help in preparing the user study. This research was supported by Next-Generation Information Computing Development Program through the National Research Foundation of Korea(NRF) funded by the Ministry of Science, ICT (2017M3C4A7068175). This work 
was also supported by Samsung Research Funding \& Incubation Center of Samsung Electronics under Project Number SRFC-IT1701-09.

\section{A SOLUTION PROGRAM (EXAMPLE 1 IN SECTION 2.1)}

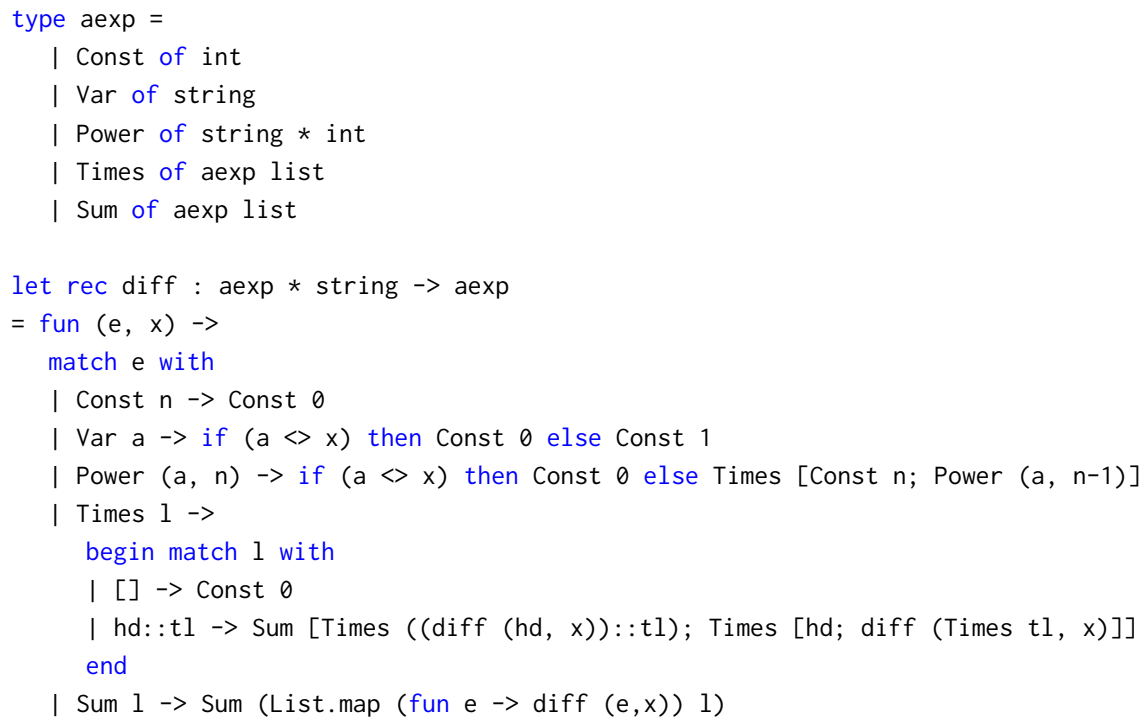

\section{B LANGUAGE}

The full language of FIxML is as follows:

$$
\begin{array}{rll}
E::= & ()|n| x \mid \text { true } \mid \text { false }|\operatorname{str}| \lambda \mathrm{x} . \mathrm{E}\left|\mathrm{E}_{1}+\mathrm{E}_{2}\right| \mathrm{E}_{1}-\mathrm{E}_{2}\left|\mathrm{E}_{1} \times \mathrm{E}_{2}\right| \mathrm{E}_{1} / \mathrm{E}_{2}\left|\mathrm{E}_{1} \bmod \mathrm{E}_{2}\right|-\mathrm{E} \\
& \text { not } E\left|E_{1}\right|\left|E_{2}\right| E_{1} \& \& E_{2}\left|E_{1}<E_{2}\right| E_{1}>E_{2}\left|E_{1} \leq E_{2}\right| E_{1} \geq E_{2}\left|E_{1}=E_{2}\right| E_{1}<>E_{2} \\
& E_{1} E_{2}\left|E_{1}:: E_{2}\right| E_{1} @ E_{2}\left|E_{1}{ }^{\wedge} E_{2}\right| \text { raise } E\left|\left(E_{1}, \ldots, E_{k}\right)\right|\left[E_{1} ; \ldots ; E_{k}\right] \\
& \text { if } E_{1} E_{2} E_{3}\left|c\left(E_{1}, \ldots, E_{k}\right)\right| \text { let } x=E_{1} \text { in } E_{2} \mid \text { let rec } f(x)=E_{1} \text { in } E_{2} \\
& \text { let } x_{1}=E_{1} \text { and } \ldots \text { and } x_{k}=E_{k} \text { in } E \mid \text { let rec } f_{1}\left(x_{1}\right)=E_{1} \text { and } \ldots \text { and } f_{k}\left(x_{k}\right)=E_{k} \text { in } E \\
& \text { match } E \text { with } p_{1} \rightarrow E_{1}|\cdots| p_{k} \rightarrow E_{k} \\
& \square \\
\tau::= & \text { unit } \mid \text { int } \mid \text { bool } \mid \text { string } \mid \text { exn }\left|\tau_{1} \rightarrow \tau_{2}\right| \tau \text { list }|T|\left(\tau_{1} * \ldots * \tau_{k}\right) \mid \alpha \\
p::= & n|x| \text { true } \mid \text { false }\left|p_{1}:: p_{2}\right|\left[p_{1} ; \ldots ; p_{k}\right]\left|\left(p_{1}, \ldots, p_{2}\right)\right| c\left(p_{1}, \ldots, p_{k}\right)\left|p_{1}\right| \cdots\left|p_{k}\right|
\end{array}
$$

\section{FEEDBACK ON A LARGE PROGRAM}

FixML was able to accurately identify and fix the error at line 103 in the following student program for problem \#13 in 3.4 seconds. Although the implementation was on a program with 154 lines, we shorten it for better readability.

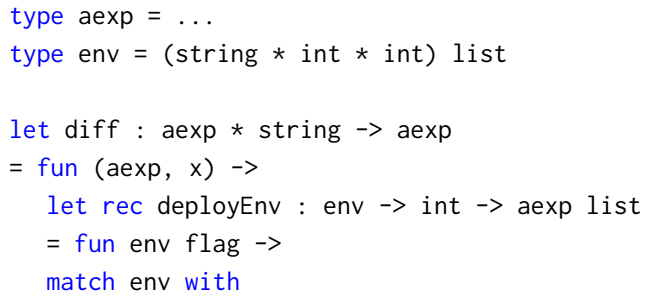




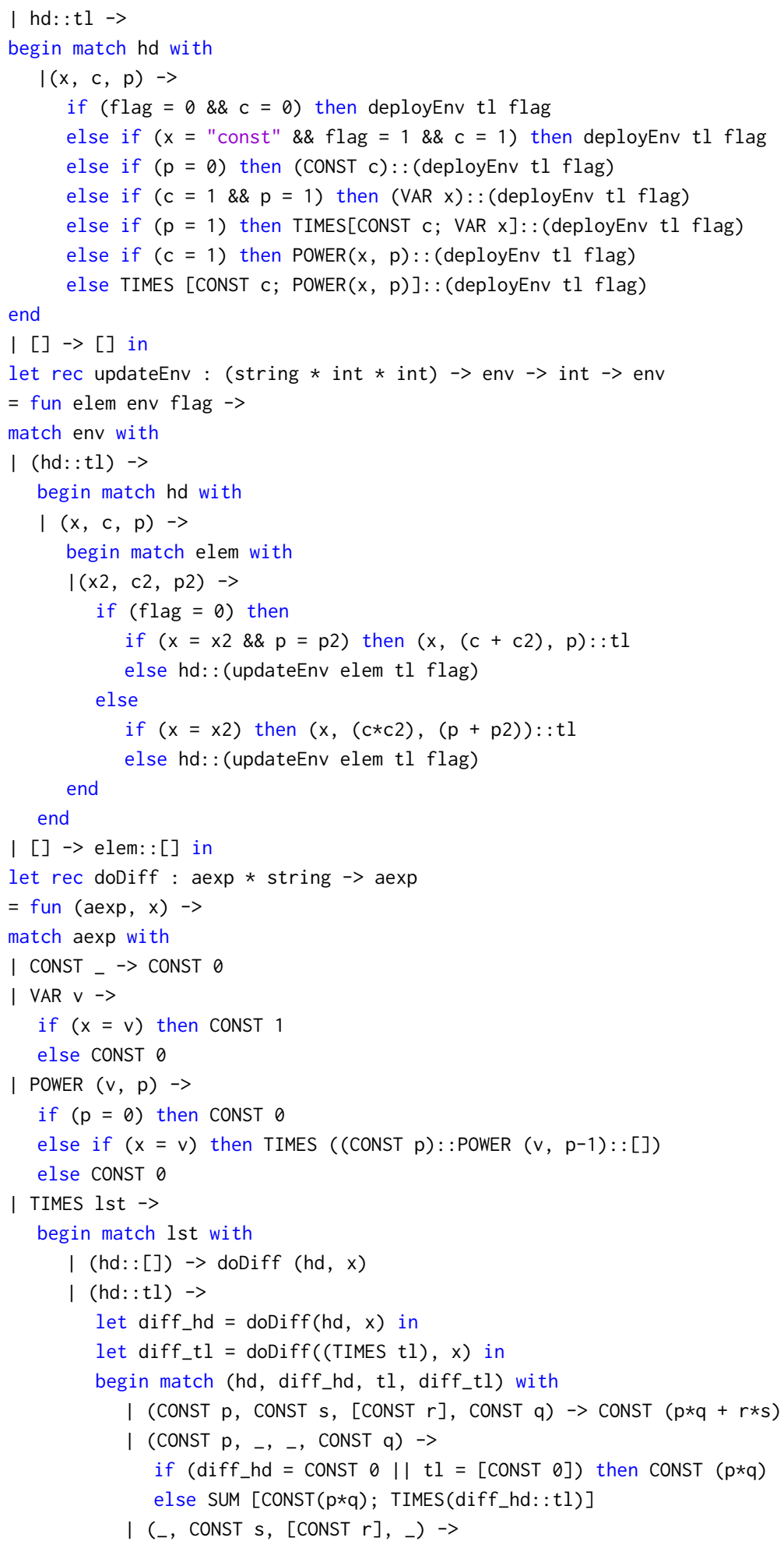




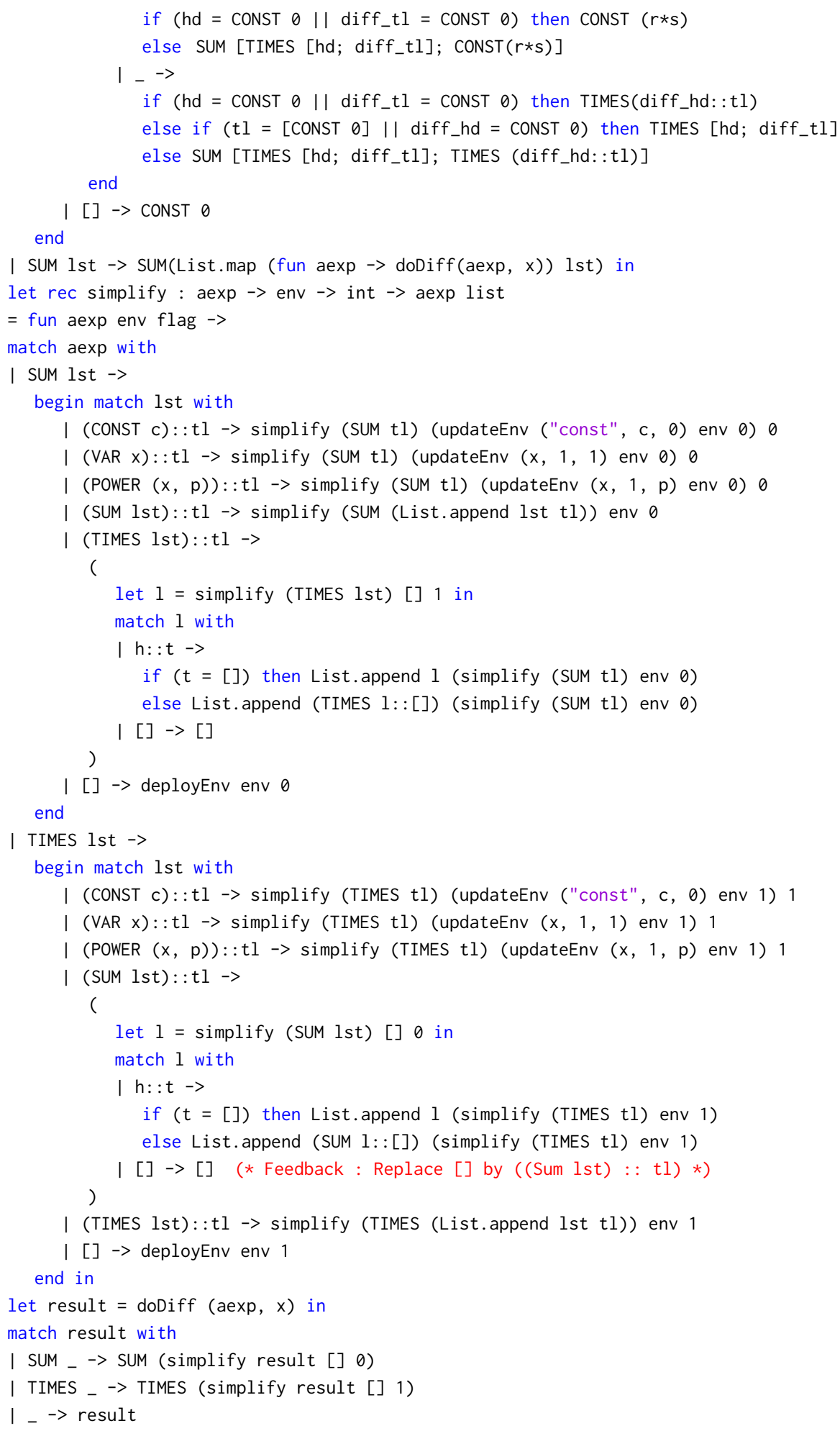




\section{REFERENCES}

Aws Albarghouthi, Sumit Gulwani, and Zachary Kincaid. 2013. Recursive Program Synthesis. In Proceedings of the 25th International Conference on Computer Aided Verification (CAV'13). Springer-Verlag, Berlin, Heidelberg, 934-950. D0I : http://dx.doi.org/10.1007/978-3-642-39799-8_67

Thomas Ball, Mayur Naik, and Sriram K. Rajamani. 2003. From Symptom to Cause: Localizing Errors in Counterexample Traces. In Proceedings of the 30th ACM SIGPLAN-SIGACT Symposium on Principles of Programming Languages (POPL '03) ACM, New York, NY, USA, 97-105. DOI : http://dx.doi.org/10.1145/604131.604140

Matej Balog, Alexander L. Gaunt, Marc Brockschmidt, Sebastian Nowozin, and Daniel Tarlow. 2017. DeepCoder: Learning to Write Programs. In ICLR.

Sahil Bhatia, Pushmeet Kohli, and Rishabh Singh. 2018. Neuro-symbolic Program Corrector for Introductory Programming Assignments. In Proceedings of the 40th International Conference on Software Engineering (ICSE '18). ACM, New York, NY, USA, 60-70. DOI : http://dx.doi.org/10.1145/3180155.3180219

Sheng Chen and Martin Erwig. 2014. Counter-factual Typing for Debugging Type Errors. In Proceedings of the 41st ACM SIGPLAN-SIGACT Symposium on Principles of Programming Languages (POPL '14). ACM, New York, NY, USA, 583-594. DOI : http://dx.doi.org/10.1145/2535838.2535863

Loris D’Antoni, Roopsha Samanta, and Rishabh Singh. 2016. Qlose: Program Repair with Quantiative Objectives. (July 2016) https://www.microsoft.com/en-us/research/publication/qlose-program-repair-with-quantiative-objectives/

Yu Feng, Ruben Martins, Jacob Van Geffen, Isil Dillig, and Swarat Chaudhuri. 2017a. Component-based Synthesis of Table Consolidation and Transformation Tasks from Examples. In Proceedings of the 38th ACM SIGPLAN Conference on Programming Language Design and Implementation (PLDI 2017). ACM, New York, NY, USA, 422-436. DOI: http: //dx.doi.org/10.1145/3062341.3062351

Yu Feng, Ruben Martins, Yuepeng Wang, Isil Dillig, and Thomas W. Reps. 2017b. Component-based Synthesis for Complex APIs. In Proceedings of the 44th ACM SIGPLAN Symposium on Principles of Programming Languages (POPL 2017). ACM, New York, NY, USA, 599-612. DOI : http://dx.doi.org/10.1145/3009837.3009851

John K. Feser, Swarat Chaudhuri, and Isil Dillig. 2015. Synthesizing Data Structure Transformations from Input-output Examples. In Proceedings of the 36th ACM SIGPLAN Conference on Programming Language Design and Implementation (PLDI '15). ACM, New York, NY, USA, 229-239. DOI : http://dx.doi.org/10.1145/2737924.2737977

Stephanie Forrest, ThanhVu Nguyen, Westley Weimer, and Claire Le Goues. 2009. A Genetic Programming Approach to Automated Software Repair. In Proceedings of the 11th Annual Conference on Genetic and Evolutionary Computation (GECCO '09). ACM, New York, NY, USA, 947-954. DOI : http://dx.doi.org/10.1145/1569901.1570031

Jonathan Frankle, Peter-Michael Osera, David Walker, and Steve Zdancewic. 2016. Example-directed Synthesis: A Typetheoretic Interpretation. In Proceedings of the 43rd Annual ACM SIGPLAN-SIGACT Symposium on Principles of Programming Languages (POPL '16). ACM, New York, NY, USA, 802-815. DOI : http://dx.doi.org/10.1145/2837614.2837629

Andreas Griesmayer, Stefan Staber, and Roderick Bloem. 2007. Automated Fault Localization for C Programs. Electron. Notes Theor. Comput. Sci. 174, 4 (May 2007), 95-111. DOI : http://dx.doi.org/10.1016/j.entcs.2006.12.032

Alex Groce, Sagar Chaki, Daniel Kroening, and Ofer Strichman. 2006. Error Explanation with Distance Metrics. Int. F. Softw. Tools Technol. Transf. 8, 3 (June 2006), 229-247. DOI : http://dx.doi.org/10.1007/s10009-005-0202-0

Sumit Gulwani. 2011. Automating String Processing in Spreadsheets Using Input-output Examples. In Proceedings of the 38th Annual ACM SIGPLAN-SIGACT Symposium on Principles of Programming Languages (POPL '11). ACM, New York, NY, USA, 317-330. DOI : http://dx.doi.org/10.1145/1926385.1926423

Sumit Gulwani, Ivan Radiček, and Florian Zuleger. 2014. Feedback Generation for Performance Problems in Introductory Programming Assignments. In Proceedings of the 22Nd ACM SIGSOFT International Symposium on Foundations of Software Engineering (FSE 2014). ACM, New York, NY, USA, 41-51. DOI : http://dx.doi.org/10.1145/2635868.2635912

Sumit Gulwani, Ivan Radiček, and Florian Zuleger. 2018. Automated Clustering and Program Repair for Introductory Programming Assignments. In Proceedings of the 39th ACM SIGPLAN Conference on Programming Language Design and Implementation (PLDI 2018). ACM, New York, NY, USA, 465-480. DOI : http://dx.doi.org/10.1145/3192366.3192387

Rahul Gupta, Soham Pal, Aditya Kanade, and Shirish K. Shevade. 2017. DeepFix: Fixing Common C Language Errors by Deep Learning. In Proceedings of the Thirty-First AAAI Conference on Artificial Intelligence, February 4-9, 2017, San Francisco, California, USA. 1345-1351. http://aaai.org/ocs/index.php/AAAI/AAAI17/paper/view/14603

James A. Jones, Mary Jean Harrold, and John Stasko. 2002. Visualization of Test Information to Assist Fault Localization. In Proceedings of the 24th International Conference on Software Engineering (ICSE '02). ACM, New York, NY, USA, 467-477. DOI : http://dx.doi.org/10.1145/581339.581397

Manu Jose and Rupak Majumdar. 2011. Cause Clue Clauses: Error Localization Using Maximum Satisfiability. SIGPLAN Not. 46, 6 (June 2011), 437-446. DOI : http://dx.doi.org/10.1145/1993316.1993550

Dohyeong Kim, Yonghwi Kwon, Peng Liu, I. Luk Kim, David Mitchel Perry, Xiangyu Zhang, and Gustavo Rodriguez-Rivera. 2016. Apex: Automatic Programming Assignment Error Explanation. SIGPLAN Not. 51, 10 (Oct. 2016), 311-327. DOI : http://dx.doi.org/10.1145/3022671.2984031

Proc. ACM Program. Lang., Vol. 2, No. OOPSLA, Article 158. Publication date: November 2018. 
Dongsun Kim, Jaechang Nam, Jaewoo Song, and Sunghun Kim. 2013. Automatic Patch Generation Learned from Humanwritten Patches. In Proceedings of the 2013 International Conference on Software Engineering (ICSE '13). IEEE Press, Piscataway, NJ, USA, 802-811. http://dl.acm.org/citation.cfm?id=2486788.2486893

Dileep Kini and Sumit Gulwani. 2015. FlashNormalize: Programming by Examples for Text Normalization. In Proceedings of the 24th International Conference on Artificial Intelligence (IfCAI'15). AAAI Press, 776-783. http://dl.acm.org/citation. cfm?id=2832249.2832357

Etienne Kneuss, Manos Koukoutos, and Viktor Kuncak. 2015. Deductive Program Repair. In Computer Aided Verification, Daniel Kroening and Corina S. Păsăreanu (Eds.). Springer International Publishing, Cham, 217-233.

Etienne Kneuss, Ivan Kuraj, Viktor Kuncak, and Philippe Suter. 2013. Synthesis Modulo Recursive Functions. SIGPLAN Not. 48, 10 (Oct. 2013), 407-426. DOI : http://dx.doi.org/10.1145/2544173.2509555

Robert Könighofer and Roderick Bloem. 2011. Automated Error Localization and Correction for Imperative Programs. In Proceedings of the International Conference on Formal Methods in Computer-Aided Design (FMCAD '11). FMCAD Inc, Austin, TX, 91-100. http://dl.acm.org/citation.cfm?id=2157654.2157671

Claire Le Goues, Michael Dewey-Vogt, Stephanie Forrest, and Westley Weimer. 2012. A Systematic Study of Automated Program Repair: Fixing 55 out of 105 Bugs for \$8 Each. In Proceedings of the 34th International Conference on Software Engineering (ICSE '12). IEEE Press, Piscataway, NJ, USA, 3-13. http://dl.acm.org/citation.cfm?id=2337223.2337225

Benjamin S. Lerner, Matthew Flower, Dan Grossman, and Craig Chambers. 2007. Searching for Type-error Messages. In Proceedings of the 28th ACM SIGPLAN Conference on Programming Language Design and Implementation (PLDI '07). ACM, New York, NY, USA, 425-434. DOI : http://dx.doi.org/10.1145/1250734.1250783

Fan Long and Martin Rinard. 2016. Automatic Patch Generation by Learning Correct Code. SIGPLAN Not. 51, 1 (Jan. 2016), 298-312. DOI : http://dx.doi.org/10.1145/2914770.2837617

Hoang Duong Thien Nguyen, Dawei Qi, Abhik Roychoudhury, and Satish Chandra. 2013. SemFix: Program Repair via Semantic Analysis. In Proceedings of the 2013 International Conference on Software Engineering (ICSE '13). IEEE Press, Piscataway, NJ, USA, 772-781. http://dl.acm.org/citation.cfm?id=2486788.2486890

Peter-Michael Osera and Steve Zdancewic. 2015. Type-and-example-directed Program Synthesis. In Proceedings of the 36th ACM SIGPLAN Conference on Programming Language Design and Implementation (PLDI '15). ACM, New York, NY, USA, 619-630. DOI : http://dx.doi.org/10.1145/2737924.2738007

Zvonimir Pavlinovic, Tim King, and Thomas Wies. 2014. Finding Minimum Type Error Sources. SIGPLAN Not. 49, 10 (Oct. 2014), 525-542. DOI : http://dx.doi.org/10.1145/2714064.2660230

Zvonimir Pavlinovic, Tim King, and Thomas Wies. 2015. Practical SMT-based Type Error Localization. SIGPLAN Not. 50, 9 (Aug. 2015), 412-423. DOI : http://dx.doi.org/10.1145/2858949.2784765

Nadia Polikarpova, Ivan Kuraj, and Armando Solar-Lezama. 2016. Program Synthesis from Polymorphic Refinement Types. In Proceedings of the 37th ACM SIGPLAN Conference on Programming Language Design and Implementation (PLDI '16). ACM, New York, NY, USA, 522-538. DOI : http://dx.doi.org/10.1145/2908080.2908093

Yewen Pu, Karthik Narasimhan, Armando Solar-Lezama, and Regina Barzilay. 2016. Sk_P: A Neural Program Corrector for MOOCs. In Companion Proceedings of the 2016 ACM SIGPLAN International Conference on Systems, Programming, Languages and Applications: Software for Humanity (SPLASH Companion 2016). ACM, New York, NY, USA, 39-40. DOI : http://dx.doi.org/10.1145/2984043.2989222

Manos Renieris and Steven P. Reiss. 2003. Fault Localization with Nearest Neighbor Queries. In Proceedings of the 18th IEEE International Conference on Automated Software Engineering (ASE'03). IEEE Press, Piscataway, NJ, USA, 30-39. DOI : http://dx.doi.org/10.1109/ASE.2003.1240292

Saul Schleimer, Daniel S. Wilkerson, and Alex Aiken. 2003. Winnowing: Local Algorithms for Document Fingerprinting. In Proceedings of the 2003 ACM SIGMOD International Conference on Management of Data (SIGMOD '03). ACM, New York, NY, USA, 76-85. DOI : http://dx.doi.org/10.1145/872757.872770

Eric L. Seidel, Huma Sibghat, Kamalika Chaudhuri, Westley Weimer, and Ranjit Jhala. 2017. Learning to Blame: Localizing Novice Type Errors with Data-Driven Diagnosis. CoRR abs/1708.07583 (2017). arXiv:1708.07583 http://arxiv.org/abs/1708. 07583

Rishabh Singh, Sumit Gulwani, and Armando Solar-Lezama. 2013. Automated Feedback Generation for Introductory Programming Assignments. SIGPLAN Not. 48, 6 (June 2013), 15-26. DOI : http://dx.doi.org/10.1145/2499370.2462195

Edward K. Smith, Earl T. Barr, Claire Le Goues, and Yuriy Brun. 2015. Is the Cure Worse Than the Disease? Overfitting in Automated Program Repair. In Proceedings of the 2015 10th foint Meeting on Foundations of Software Engineering (ESEC/FSE 2015). ACM, New York, NY, USA, 532-543. DOI : http://dx.doi.org/10.1145/2786805.2786825

Sunbeom So and Hakjoo Oh. 2017. Synthesizing Imperative Programs from Examples Guided by Static Analysis. In Static Analysis - 24th International Symposium, SAS 2017, New York, NY, USA, August 30 - September 1, 2017, Proceedings. 364-381. DOI : http://dx.doi.org/10.1007/978-3-319-66706-5_18

Ke Wang, Rishabh Singh, and Zhendong Su. 2018. Search, Align, and Repair: Data-driven Feedback Generation for Introductory Programming Exercises. In Proceedings of the 39th ACM SIGPLAN Conference on Programming Language 
Design and Implementation (PLDI 2018). ACM, New York, NY, USA, 481-495. DOI : http://dx.doi.org/10.1145/3192366. 3192384

Westley Weimer, ThanhVu Nguyen, Claire Le Goues, and Stephanie Forrest. 2009. Automatically Finding Patches Using Genetic Programming. In Proceedings of the 31st International Conference on Software Engineering (ICSE '09). IEEE Computer Society, Washington, DC, USA, 364-374. DOI : http://dx.doi.org/10.1109/ICSE.2009.5070536

Baijun Wu, John Peter Campora III, and Sheng Chen. 2017. Learning User Friendly Type-error Messages. Proc. ACM Program. Lang. 1, OOPSLA, Article 106 (Oct. 2017), 29 pages. DOI : http://dx.doi.org/10.1145/3133930

Baijun Wu and Sheng Chen. 2017. How Type Errors Were Fixed and What Students Did? Proc. ACM Program. Lang. 1, OOPSLA, Article 105 (Oct. 2017), 27 pages. DOI : http://dx.doi.org/10.1145/3133929

Navid Yaghmazadeh, Yuepeng Wang, Isil Dillig, and Thomas Dillig. 2017. SQLizer: Query Synthesis from Natural Language. Proc. ACM Program. Lang. 1, OOPSLA, Article 63 (Oct. 2017), 26 pages. DOI : http://dx.doi.org/10.1145/3133887

Danfeng Zhang, Andrew C. Myers, Dimitrios Vytiniotis, and Simon Peyton-Jones. 2017. SHErrLoc: A Static Holistic Error Locator. ACM Trans. Program. Lang. Syst. 39, 4, Article 18 (Aug. 2017), 47 pages. DOI : http://dx.doi.org/10.1145/3121137 